\title{
High-Efficiency Semiconductor Resonant-Cavity Light-Emitting Diodes: A Review
}

\author{
Danaë Delbeke, Student Member, IEEE, Ronny Bockstaele, Member, IEEE, Peter Bienstman, Member, IEEE, \\ Roel Baets, Senior Member, IEEE, and Henri Benisty
}

Invited Paper

\begin{abstract}
An overview of highly efficient resonant-cavity lightemitting diodes is presented. First, the basics of dipole emission in planar cavities are reviewed. From these, a number of design rules are derived. We point out some guidelines for comparison of high-efficiency light-emitting diodes, and use these to review the state-of-the-art devices in different material systems and at different wavelengths. We also discuss some advanced techniques based on gratings or photonic crystals to improve the efficiency of these devices.
\end{abstract}

Index Terms-High efficiency, light-emitting diode, microcavity, resonant-cavity, spontaneous emission.

\section{INTRODUCTION}

$\mathbf{T}$ HE EXPLORATORY development of the light-emitting diode (LED) started with first reports of electroluminescence by Round (1907) and Losev (1923), and continued with the understanding of recombination phenomena by Lehovec (1951) and the first fabrication of an infrared LED at MIT (1960). During the mid sixties or the early handheld calculator epoch, research accelerated [1]. Since then, the development of the cold light sources has been characterized by a race for high efficiency. This optimization toward a higher efficiency took more or less place in three stages, reflected by a change in focus to increase the efficiency.

The external quantum efficiency $\eta_{\text {ext }}$ is the key performance figure for high-efficiency LEDs and stands for the number of photons generated per injected electron

$$
\eta_{\text {ext }}=\frac{P_{\mathrm{opt}} / \hbar \omega}{I / q}=\eta_{\mathrm{inj}} \eta_{\mathrm{rad}} \eta_{\text {extr }}
$$

with $P_{\text {opt }}$ the emitted optical power (W), $I$ the injection current (A), $q$ the elementary electron charge (C) and $\hbar \omega$ the en-

Manuscript received January 7, 2002; revised February 7, 2002. This work was supported in part by the European Union Esprit under Program SMILES/SMILED (Semiconductor Microcavity Light Emitting Diodes) and under Program Belgian Inter-University Attraction Pole IAP IV-13. D. Delbeke was supported by IWT (Institute for the Promotion of Innovation by Science and Technology in Flanders). P. Bienstman was supported by the Flemish Fund for Scientific Research (FWO Vlaanderen) under a postdoctoral fellowship.

D. Delbeke, R. Bockstaele, P. Bienstman, and R. Baets are with the Department of Information Technology (INTEC), Ghent University-IMEC, B-9000 Gent, Belgium.

H. Benisty is with the Laboratoire de Physique de la Matière Condensée, Ecole Polytechnique, F-91128 Palaiseau Cedex, France.

Publisher Item Identifier S 1077-260X(02)03777-2. ergy per emitted photon $(\mathrm{eV}) \cdot \eta_{\text {ext }}$ depends on the fraction of carriers injected in the useful active region $\left(\eta_{\mathrm{inj}}\right)$, the fraction of spontaneous recombination that is radiative $\left(\eta_{\text {rad }}\right)$ and the extraction efficiency of the generated photons $\left(\eta_{\text {extr }}\right)$. These three contributions are representative for the more or less successive introductions of optimization. Firstly, exhaustive study of semiconductor electronic band structures in the early sixties led to a better control of carrier injection. Accordingly, $\eta_{\text {inj }}$ could be raised, reaching unity (1963 [2]) by the introduction of heterostructures and quantum wells (QWs). The introduction of liquid phase epitaxy (LPE) (1967), metal-organic chemical vapor deposition (MOCVD), and molecular beam epitaxy (MBE) (1980) overcame the moderate $\eta_{\text {rad }}-$ values. Non radiative recombination at low current densities mainly originates from defects in the crystal. Successive improvement of epitaxial fabrication techniques resulted in the availability of high-quality crystals. Nowadays, the quality of the crystal measured by the density of defects, can be very high for a broad range of material systems. For AlInGaAs on GaAs substrates for example, this results in an $\eta_{\text {rad }}$ reaching unity. However, even today the internal efficiency remains a major issue for some material systems, e.g., in AlInGaP LEDs and in LED structures in which substantial reabsorption occurs.

It is at this stage $\left(\eta_{\text {inj }} \eta_{\text {rad }} \approx 1\right)$ that it is appropriate to optimize $\eta_{\text {extr }}$ which is limited to $2-4 \%$ by Snell's law for conventional planar LEDs due to the high refractive index contrast between the source material and the surrounding medium. This optimization happened in the early nineties, when cavity optics entered the world of LEDs with the resonant-cavity LED (RCLED). In these devices, the active layer is embedded in a cavity with at least one dimension of the order of the wavelength of the emitted light. Under those circumstances, the spontaneous emission process itself is modified, such that the internal emission is no longer isotropic. In spite of earlier work by Lukosz on emission in multilayers in 1980 [3]-[6], experimental evidence has only been introduced in 1990-1992 by the work of Yokoyama, Björk, Yamamoto, Deppe, Hunt et al. [7]-[13], Schubert et al. presented the RCLED as a conceptual novel LED in 1992 [14]. In 1994, a communication application design has been proposed by the same group [15]. However, the overall external efficiency remained low. The first highly efficient RCLED was made by De Neve et al. in 
1995 obtaining $\eta_{\text {ext }} \approx 23 \%$ [16]-[18], and has been further optimized in 1999 by Wierer et al. $\left(\eta_{\text {ext }} \approx 27 \%\right)$ [19]. Parallel with this "wave" optics-based technique exploiting cavity interference effects, "ray" optics-based techniques oriented toward geometrical light collection were introduced. In 1992, Hewlett-Packard produced its first LED with transparent superstrate $\left(\eta_{\text {ext }} \approx 6 \%\right)$. Elaboration of the principle and geometrical optimization resulted in 1999 in a pyramidal shaped device with world record efficiencies of 60\% [20]. In 1993, Schnitzer and Yablonovitch [21] proposed the idea of random surface texturing to enhance escape probability by scattering of the photons, a principle resumed in 1998 by Windisch et $a l$. giving rise to thin film devices with $\eta_{\text {ext }} \approx 50 \%$ [22]. The LED with a radial outcoupling taper [23] is another successful technique which tilts the path of the photon inside the semiconductor until it reaches the semiconductor-air interface within the extraction cone.

This paper will give a review of the RCLED. This high-efficiency light emitter is no longer just an object of research. It has been launched commercially in 1999 by Mitel Corp. (now Zarlink Semiconductors) for plastic optical fiber (POF) communication applications. The market for LEDs for communications is increasing, for instance the newly approved standard for 100BASE-SX (Fast Ethernet) is based on 850-nm LEDs [24]. RCLEDs are benefitting from this evolution: recently, RCLEDs emitting red light in combination with POF is proposed as the standard for FireWire or i.Link (IEEE1394b) [25]. This standard covers broadband service applications such as digital TV, DVD, etc. Several companies do participate in this commercialization of the RCLED for communication applications and some extend this activity toward noncommunication applications.

First, a theoretical investigation of the planar RCLED is presented. Theoretical models to analyze spontaneous emission can mainly be subdivided in two major approaches. The first series is a quantum-mechanical description based on quantization of the electromagnetic field and mode density. These methods can become complex and their application is restricted to simple, ideal media [9], [26]. The second series is based on a classical approach valid in the so-called weak-coupling regime, which is the working regime of practical planar RCLEDs. Spontaneous emission is modeled as an electric dipole. To calculate the far field emission, a transfer matrix formalism is implemented for the plane wave components of the dipole field. This numerical method is described in detail in a series articles by $\mathrm{H}$. Benisty et al. [27]-[29] and in [30], [31]. For the sake of physical insight, this numerically solvable analysis is summarized in Section II. The different parameters influencing $\eta_{\text {extr }}$ are then presented in Section III. Section IV discusses some guidelines and figures of merit for comparison of high-efficiency LEDs. Section $\mathrm{V}$ reviews the state-of-the-art of high-efficiency RCLEDs. Although planar RCLEDs have been optimized thoroughly, advanced techniques to improve $\eta_{\text {extr }}$ even further are nevertheless still welcome. Some of these techniques are presented in Section VI. Future prospects end this paper.

Although the principle is extendable to all sorts of material, the examples and general trends given in this paper are limited to semiconductor devices.
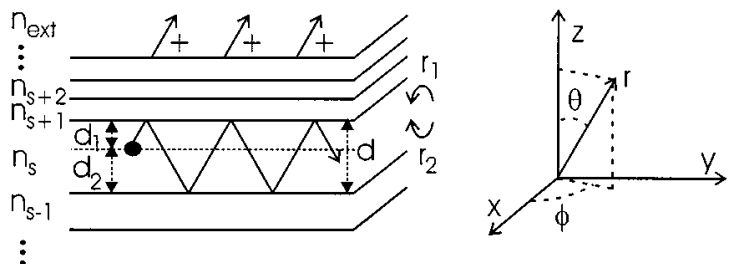

Fig. 1. Structure of a light-emitting multilayer device. The emitting medium has a refractive index $n_{s}$, the intermediate layers $n_{s \pm 1}, n_{s \pm 2}, \ldots$, the half infinite surrounding media $n_{\text {cxt }}$. The emitting layer has a thickness $d$. The dipole is located at a distance $d_{1}$ from the interface with the first layer of the upper mirror; at a distance $d_{2}$ from the interface with the first layer of the bottom mirror. The upper mirror has a reflection coefficient $r_{1}$; the bottom mirror a reflection coefficient $r_{2}$. Interference takes place when the radiation is reflected back and forth between the two interfaces of the layer. The emission angle $\theta$ and $\phi$ are defined in the $x y z$ coordinate system.

\section{BASICS OF SPONTANEOUS EMISSION IN A LAYERED MEDIUM}

\section{A. Modification of Emission Pattern: Theoretical Treatment}

In an RCLED [also called micro-cavity LED (MCLED)], spontaneous emission is generated in a multilayer Fabry-Pérot (FP) resonator, in which interference effects alter the internal angular power distribution (Fig. 1). With an appropriate cavity design, the preferential propagation direction of the photons can thus be forced from total internal reflection regime toward the extraction cone, benefitting to $\eta_{\text {extr }}$. Together with this increase of directivity and/or efficiency due to a redistribution of the photons, the spontaneous emission rate will be enhanced due to the Purcell effect. However, because of the planar geometry and the rather small reflectivity coefficients of the cavity mirror(s) in practical applications, the Purcell-factor is close to one, resulting in a negligible spontaneous emission rate enhancement (see Section II.B).

In this so-called "weak coupling" regime, the spontaneous emission of electron/hole pairs can adequately be represented by an electric dipole. An electric dipole can be decomposed in a horizontal electric dipole (with dipole moment in the $(x-y$ )-plane) and a vertical electric dipole (with dipole moment along $z$ ). In bulk semiconductor material the dipoles can have any orientation or equivalently one third of the power generated by the dipoles is generated by vertical dipoles and two thirds by horizontal dipoles. In unstrained QWs there is a substantial preference for emission through horizontal dipoles [33]. Furthermore, if a compressive strain is added to the $\mathrm{QW}$, a strong enhancement of radiation through horizontal dipoles is realized, while tensile strain enhances vertical dipoles [34]. The normalized monochromatic electromagnetic field from the dipole $\boldsymbol{\Psi}_{\text {dip }}(x, y, z)$ can be Fourier transformed with respect to $x$ and $y$ in a set of plane waves [30]. The $\mathbf{k}_{||}$Fourier spectrum contains arbitrarily large wave vectors $\mathbf{k}_{x}$ and $\mathbf{k}_{y}$, spanning propagative $\left(\left|\mathbf{k}_{\|}\right| \leq n_{s} k_{0}\right)$ and evanescent contributions $\left(\left|\mathbf{k}_{\|}\right|>n_{s} k_{0}\right)$, with $n_{s}$ the refractive index of the source layer, $k_{0}=2 \pi / \lambda_{0}$ and $\lambda_{0}$ the vacuum wavelength and $\mathbf{k}_{\|}=\mathbf{k}_{x}+\mathbf{k}_{y}$. The $z$-component of $\mathbf{k}, k_{z}$, can be expressed as a function of $k_{\|}$

$$
k_{z}= \pm \sqrt{n_{s}^{2} k_{0}^{2}-k_{x}^{2}-k_{y}^{2}}= \pm \sqrt{n_{s}^{2} k_{0}^{2}-k_{\|}^{2}} .
$$



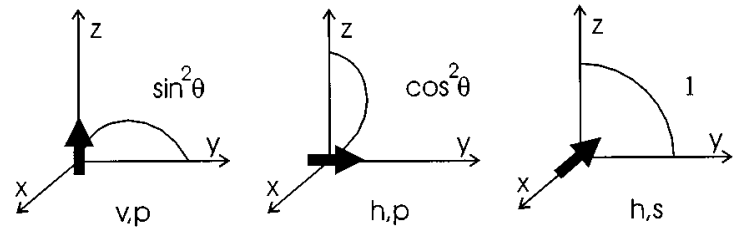

Fig. 2. Emission pattern of dipoles in bulk media (a) vertical dipole emitting TM. (b) Horizontal dipole emitting TM. (c) Horizontal dipole emitting TE.

TABLE I

SOURCE TERMS FOR HorizonTAL AND VerTiCAL Dipoles

\begin{tabular}{c||c|c}
\hline & TE & TM \\
\hline \hline Horizontal & $A_{\uparrow \downarrow}^{h, s}=\sqrt{\frac{3}{16 \pi}}$ & $A_{\uparrow \downarrow}^{h, p}=\sqrt{\frac{3}{16 \pi} \frac{k_{z}}{k}}$ \\
\hline Vertical & $A_{\uparrow \downarrow}^{v, s}=0$ & $A_{\uparrow \downarrow}^{v, p}= \pm \sqrt{\frac{3}{8 \pi}} \frac{k_{\|}}{k}$ \\
\hline
\end{tabular}

Arranging the integrand of the Fourier transformation properly, the plane wave amplitude $A=|\mathbf{A}|$ can be expressed as a density per unit solid angle $d \Omega=\sin \theta d \theta d \varphi$

$$
\begin{aligned}
\Psi_{\text {dip }}(x, y, z) & \int_{-\infty}^{+\infty} \int_{-\infty}^{+\infty} \mathbf{A}\left(k_{x}, k_{y}\right) \exp \left(-j \mathbf{k} \cdot \mathbf{r}_{|z|}\right) \frac{d k_{x} d k_{y}}{n_{s} k_{0} k_{z}} \\
= & \left(\int_{0}^{\pi / 2}+\int_{\pi / 2+j 0}^{\pi / 2+j \infty}\right) \int_{0}^{2 \pi} \mathbf{A}(\theta, \varphi) \\
& \quad \times \exp \left(-j \mathbf{k} \cdot \mathbf{r}_{|z|}\right) \sin \theta d \theta d \varphi
\end{aligned}
$$

where a time variation of $\exp (j \omega t)$ is assumed and in which for the square root of (2) the solution with zero or negative imaginary part is chosen and $\mathbf{r}_{|z|}=x \mathbf{1}_{x}+y \mathbf{1}_{y}+|z| \mathbf{1}_{z}$. A plane wave component $\mathbf{A}$ of the field resulting from an electric dipole has its electric field in the plane of the dipole moment and the wavevector $\mathbf{k}$, vanishing sinusoidally for emission in the direction of the dipole moment [32] (Fig. 2). Decomposing an arbitrary linear polarization into TE (transverse electric or $s$ : the plane wave has its $E$-field in the $(x-y)$-plane and orthogonal to $k$ ) and TM (transverse magnetic or $p$ : has its $H$-field transverse to the plane of incidence), averaging the field amplitude over the azimuthal angle $\varphi$ and normalizing the total emitted power (the term $1 / 2 \sqrt{\varepsilon / \mu_{0}}$ is omitted for simplicity) results in plane wave amplitudes (expressed as a density per unit solid angle) summarized in Table I.

Decomposition of the electromagnetic problem in purely TE and TM systems in relation with the multilayer interfaces, transforms the vectorial electromagnetic problem in three simple scalar problems. Indeed, the beauty of TE-TM decomposition lies in the polarization-maintaining reflection or refraction of TE and TM waves upon a planar interface. Analysis of electromagnetic wave propagation in an isotropic layered structure can as such be decomposed in two uncoupled systems. The TM system has to be elaborated for vertical and horizontal dipoles, the TE system only for horizontal dipoles. From Fig. 1, it is clear that for the respective dipole orientations and polarizations, the emitted intensity $I^{\mathrm{or}, \mathrm{pol}}(\theta)$, with $\theta$ the internal emission angle, caused by the source's plane wave component $A^{\text {or,pol }}$ is given by $($ or $=h, v ;$ pol $=s, p)$ :

$$
\begin{aligned}
I^{\mathrm{or}, \mathrm{pol}}(\theta)= & T_{1} I_{\mathrm{dip}}^{\mathrm{or}, \mathrm{pol}}(\theta) \\
I_{\mathrm{dip}}^{\mathrm{or}, \mathrm{pol}}(\theta)= & \mid\left(A_{\uparrow}^{\mathrm{or}, \mathrm{pol}}+A_{\downarrow}^{\mathrm{or}, \mathrm{pol}} r_{2} \exp \left(-j 2 \phi_{2}\right)\right) \\
& \times\left(1+r_{1} r_{2} \exp (-j 2 \phi)\right. \\
& \left.+r_{1}^{2} r_{2}^{2} \exp (-j 4 \phi)+\ldots\right)\left.\right|^{2} \\
= & \frac{\left|A_{\uparrow}^{\mathrm{or}, \mathrm{pol}}+A_{\downarrow}^{\mathrm{or}, \mathrm{pol}}\right| r_{2}\left|\exp \left(-j 2 \phi_{2 \mathrm{eff}}\right)\right|^{2}}{|1-| r_{1} r_{2}\left|\exp \left(-j 2 \phi_{\mathrm{eff}}\right)\right|^{2}}
\end{aligned}
$$

with $\left|r_{1} r_{2}\right|<1, r_{1}, r_{2}$ the upwards and downwards amplitude reflection coefficients, $T_{1}$ the upwards power transmission coefficient, $\phi_{i}=k_{0} n_{s} d_{i} \cos \theta, i=1,2, \phi=\phi_{1}+\phi_{2}$ and $2 \phi-\arg \left(r_{1}\right)-\arg \left(r_{2}\right)=2 \phi_{\mathrm{eff}}(\theta, \lambda)$ and $2 \phi_{i}-\arg \left(r_{i}\right)=$ $2 \phi_{i \text { eff }}(\theta, \lambda)$ and $d_{1}, d_{2}$ the distances of the dipole from the interfaces of, respectively, the first layer of the upper mirror and the first layer of the bottom mirror. $r_{1}, r_{2}, T_{1}$ are polarization dependent and can be calculated using a transfer matrix method [30]. The numerator is called the standing wave factor $\zeta\left(\phi_{2 \mathrm{eff}}\right)$ and is responsible for the dependence of the emitted intensity on the position of the source: the radiated emission in a particular direction is high if the standing wave field strength at the source position is high. The denominator is independent of the position of the source, but depends strongly on $\lambda$ and $\theta$, and is called the cavity enhancement factor or Airy factor $\Gamma\left(\phi_{\text {eff }}\right)$. The Airy function is periodic in $\phi_{\text {eff }}$ with a period $\pi$, and at a maximum, the cavity is said to be resonant. These maxima define a resonant mode and obey the phase condition

$$
2 \phi-\arg \left(r_{1}\right)-\arg \left(r_{2}\right)=2 \phi_{\mathrm{eff}}(\theta, \lambda)=2 m \pi
$$

with $m$ a positive or negative integer. In Section III, it will be clear that these resonances account both for FP $\left(\theta<\theta_{c}\right)$ and guided modes $\left(\theta>\theta_{c}\right)$, with $\theta_{c}=\arcsin \left(n_{\text {ext }} / n_{s}\right)$ the critical angle for total internal reflection (TIR), with $n_{\text {ext }}$ the refractive index of the external surrounding medium (see Fig. 1). If $\left|r_{1} r_{2}\right|=1$, the resonator is "perfect" and the mode will never be damped after the excitation is switched off. This occurs when there are no losses, neither due to absorption nor extraction.

For bulk semiconductor material where emitting dipoles are isotropic on average, the total emitted angular intensity $I(\theta)$ is given by

$$
I(\theta)=\frac{2}{3}\left(I^{h, s}(\theta)+I^{h, p}(\theta)\right)+\frac{1}{3} I^{v, p}(\theta)
$$

and the extraction efficiency $\eta_{\text {extr }}$ can be defined

$$
\eta_{\text {extr }}=\frac{2 \pi \int_{0}^{\theta_{c}} I(\theta) \sin \theta d \theta}{2 \pi \int_{0}^{2 \pi} I(\theta) \sin \theta d \theta} .
$$

Equation (8) has to be solved numerically, approximating the integral by a discrete summation. 


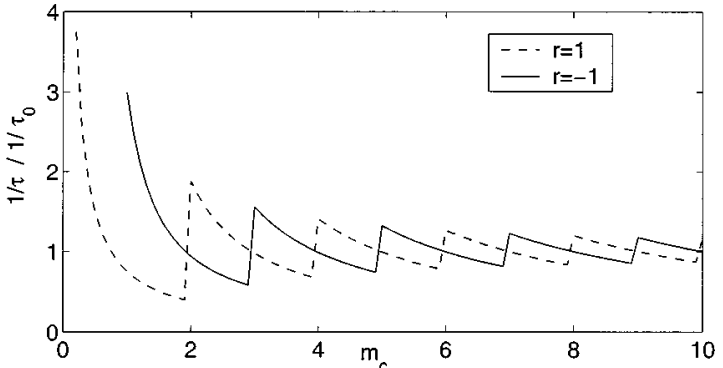

Fig. 3. Modification of spontaneous emission rate or the Purcell effect according to (10) and (11).

\section{B. Total Emission Enhancement or Purcell Factor}

The impact of the cavity on the total power emitted by the dipole can be expressed as the spontaneous emission (SE) lifetime alteration of the radiative electron-hole recombination process

$$
\frac{\frac{1}{\tau}}{\frac{1}{\tau_{0}}}=\frac{\text { emitted dipole power in cavity }}{\text { emitted dipole power in bulk }}
$$

where $\tau$ and $\tau_{0}$ are the lifetimes with and without cavity. The change of carrier lifetime due to the presence of a cavity is known as the Purcell effect [35]. The Purcell factor expresses this carrier lifetime change and has been derived by Purcell as $\left(3 Q / 4 \pi^{2}\right)\left(\lambda^{3} / V\right)$. It is defined for 3-D optical cavities of volume $V$ and mode quality factor $Q=\lambda / \delta \lambda$ with $\delta \lambda$ the narrow emission linewidth around $\lambda[36]$.

The impact of a planar cavity on the change of spontaneous decay rate has been studied in detail [8], [37], [38]. In the case of a cavity with perfect (100\% reflecting) mirrors, an analytical expression for the SE enhancement has been derived in [38] for the case of a horizontal dipole in the middle of the cavity. The results depend on the phase of the mirror reflection coefficient. For $r=+1$ there are $\left\lfloor m_{C \text { eff }}\right\rfloor=\left\lfloor m_{c}\right\rfloor+1$ modes, but only $\left\lfloor m_{c} / 2+1\right\rfloor$ are excited $(\lfloor\rfloor$ means: largest integer smaller than the argument), with $m_{c}$ the cavity order or normalized cavity length, a measure for the number of resonant modes, which is defined in Section III. The others are not excited because the dipole is located at a zero of the mode profile. The decay rate enhancement can then be expressed as

$$
\frac{\frac{1}{\tau}}{\frac{1}{\tau_{0}}}=\frac{6\left\lfloor\frac{m_{c}}{2}\right\rfloor+3}{4 m_{c}}+\frac{4\left\lfloor\frac{m_{c}}{2}\right\rfloor^{3}+6\left\lfloor\frac{m_{c}}{2}\right\rfloor^{2}+2\left\lfloor\frac{m_{c}}{2}\right\rfloor}{2 m_{c}^{3}} .
$$

For $r=-1$ there are $\left\lfloor m_{C \text { eff }}\right\rfloor=\left\lfloor m_{c}\right\rfloor$ modes, $\left\lfloor\left(m_{c}+1\right) / 2\right\rfloor$ of which are excited and the resulting decay rate enhancement is

$$
\frac{\frac{1}{\tau}}{\frac{1}{\tau_{0}}}=\frac{6\left\lfloor\frac{m_{c}+1}{2}\right\rfloor}{4 m_{c}}+\frac{4\left\lfloor\frac{m_{c}+1}{2}\right\rfloor^{3}-\left\lfloor\frac{m_{c}+1}{2}\right\rfloor}{2 m_{c}^{3}} .
$$

The terms in $1 / m_{c}$ in these expressions correspond to TE-waves, whereas the terms in $1 / m_{c}^{3}$ correspond to TM-waves. The two expressions are shown in Fig. 3 as a function of $m_{c}$. One can see that apart from the singular $1 / m_{c}$ behavior for small $m_{c}$ in the $r=+1$ case, the maximum SE enhancement is three and is obtained in a half-wavelength thick cavity with $r=-1$ (perfect metallic mirrors). For thick cavities the Purcell effect converges to one. In other words, thick cavities with many modes have a similar impact on the dipole as uniform space with a continuum of modes. For real metals, the phase shift lies in between these extreme cases, and can give rise to an intermediate Purcell factor.
In three-dimensional (3-D) cavities, the Purcell effect can be substantially larger than in planar cavities. In [35] a strong enhancement of the spontaneous emission rate has been observed for self-assembled InAs-GaAs quantum boxes inserted in GaAs-based pillar microcavities $(\times 5)$ and microdisks $(\times 15)$ using time-resolved as well as c.w. photoluminescence experiments, and in spite of various detrimental averaging effects compared to the ideal case.

\section{DESIGN RULES}

A quantitatively accurate design of an RCLED is only possible by a numerical analysis with the methods presented in Section II.A. However, a number of approximate design rules can be used as guidelines in the first phase of a design. If one allows for some parameter variations in the experimental devices, it is often unnecessary to move to detailed numerical analysis, at least for simple planar RCLEDs. An RCLED can be optimized to fulfill diverse conditions. We will discuss the optimization of overall extraction efficiency, considering several practical configurations. First, some cavity variables will be defined.

\section{A. Finesse, Quality Factor, and (Effective) Cavity Order}

The full-width at half-maximum (FWHM) of the resonant peaks $\delta \phi_{\text {eff }}$ caused by the Airy factor $\Gamma\left(\phi_{\mathrm{eff}}\right)$, is inversely proportional to the finesse $F$ :

$$
F \equiv \frac{\Delta \phi_{\mathrm{eff}}}{\delta \phi_{\mathrm{eff}}}=\frac{\pi}{\delta \phi_{\mathrm{eff}}}
$$

with $\Delta \phi_{\text {eff }}$ the separation between two adjacent resonances. The cavity order $m_{c}$ is defined as the normalized cavity length, when ideal mirrors are assumed $\left(\arg \left(r_{1}\right)+\arg \left(r_{2}\right)=0\right)$ :

$$
\begin{aligned}
m_{c} & \equiv \frac{d}{\lambda_{0} / 2 n_{s}} \\
& =\left.\frac{\lambda_{0}}{\pi} \frac{\partial \phi}{\partial \lambda}\right|_{\theta \approx 0} \\
& =\frac{1}{\pi} \frac{\partial \phi}{\partial(\cos \theta)} .
\end{aligned}
$$

The cavity order $m_{c}$ is a measure for the number of resonant modes in the bare ideal cavity: from reformulation of the resonance condition (6) as $m_{c} \cos \theta=m$, it is clear that the number of resonances is limited to $\left\lfloor m_{c}\right\rfloor$. It also links $F$ with the quality factor $Q$ of the FP mode, when small variations are considered

$$
\begin{aligned}
Q & \equiv \frac{\phi_{\mathrm{eff}}}{\delta \phi_{\mathrm{eff}}}=\frac{1}{\delta \cos (\theta)}=\frac{\lambda_{0}}{\delta \lambda} \\
& =\frac{\lambda_{0}}{\pi} \frac{\delta \phi_{\mathrm{eff}}}{\delta \lambda} \frac{\pi}{\delta \phi_{\mathrm{eff}}}=m_{c} F .
\end{aligned}
$$

Using the numerical analysis of Section II.A, the intensity emitted by an isotropic point source in an RCLED of cavity order $m_{c}=4$ with ideal mirrors can be calculated and is depicted in Fig. 4(a). The Airy function $\Gamma$ is modulated with the standing wave factor $\zeta$ for respective source positions s1 and s2 placed at different distances from the bottom mirror interface $d_{2}$ as defined in Fig. 4(d). This coupling strength $\zeta$ is proportional to the respective mode intensity at the source position. When the source is located at a node of the standing wave pattern, no coupling occurs. This happens for the second and forth mode 


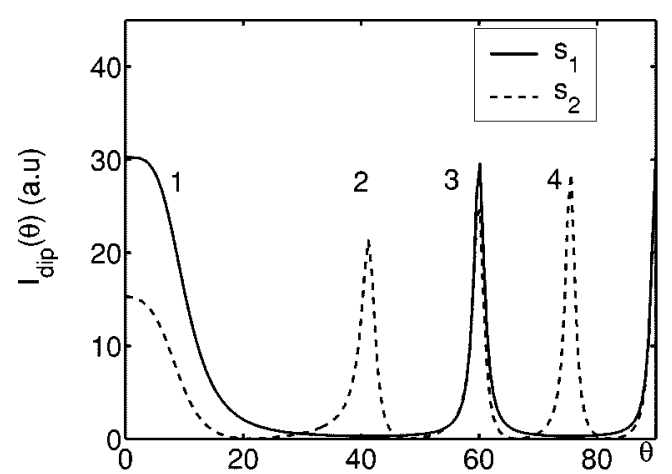

(a)

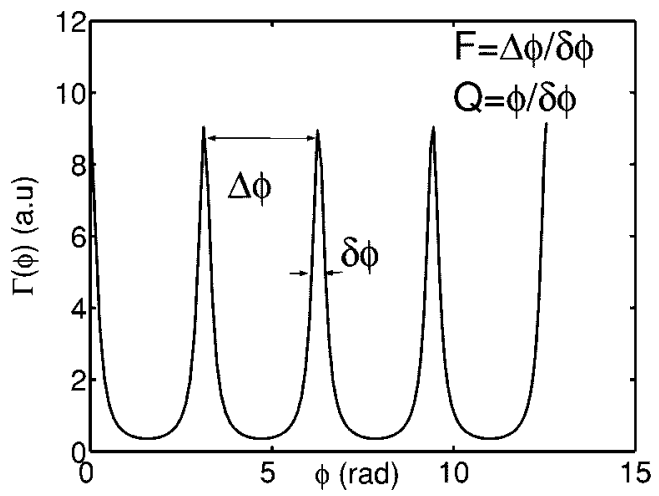

(c)

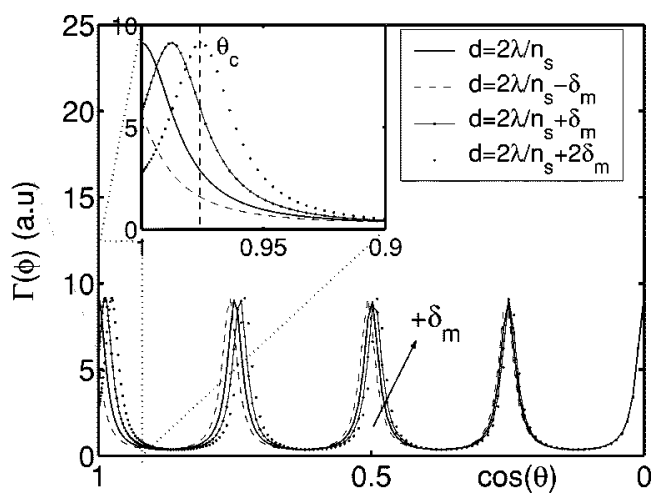

(b)

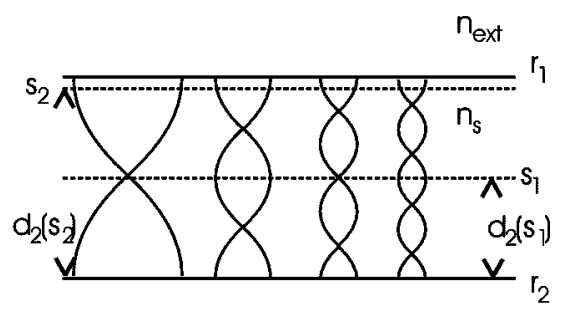

(d)

Fig. 4. (a) Internal angle-resolved TE intensity of an ideal (angle independent reflection coefficients $r_{1}$ and $r_{2}$ ) RCLED with $m_{c}=4$ for, respectively, source location s1 and s2. (b) Corresponding Airy Function of, respectively, a $\lambda$-, $\left(\lambda-\delta_{m}\right)-,\left(\lambda+\delta_{m}\right)$ - and $\left(\lambda+2 \delta_{m}\right)$-cavity for source location s1. (c) Graphical definition of $Q$ and $F$. (d) Resonant mode distributions in RCLED and source locations s1 and s2.

when the source is located at $\mathrm{s} 1$. For a dipole positioned at the height of s2, coupling to all modes is realized. The resonant mode in the extraction cone $\left(\theta<\theta_{c}\right)$ is called the FP mode, the other "trapped" modes are the guided modes. The Airy function $\Gamma$ is presented for different cavity thicknesses around $d=2 \lambda_{0} / n_{s}=2 \lambda_{s}$ in Fig. 4(b). The source is kept at the center of the cavity, leaving $\zeta$ invariable. A shift of the resonant peaks toward smaller $\cos (\theta)$ (larger $\theta$ ) for increasing cavity thickness is observed. An optimal value for $\delta_{m}$ is deduced in the next paragraph. $Q$ and $F$ are graphically defined in Fig. 4(c).

In reality, the phase of the reflection coefficients $r_{1}$ and $r_{2}$ of a multilayer depends on the wavelength and angle of incidence. Considering the FP mode, in a small range around its working point, the phase will change approximatively in a linear way. This allows to define penetration depth, defined as the depth measured from the interface, where an ideal mirror interface should be positioned to give rise to the same variation of the phase. As the reflectivities of the individual interfaces have a distinct spectral and angular behavior, two penetration depths and corresponding effective cavity thicknesses can be defined using (6) and (13)-(15)

$$
\begin{aligned}
d_{\mathrm{eff}, \theta} & =d+d_{\text {pen }, \theta} \\
& =d+\frac{\lambda_{0}}{2 n_{s}} \frac{-\partial\left(\arg \left(r_{1}\right)+\arg \left(r_{2}\right)\right)}{\partial \cos \theta} \frac{1}{2 \pi} \\
\left.d_{\mathrm{eff}, \lambda}\right|_{\theta \approx 0} & =d+d_{\text {pen }, \lambda} \\
& =d+\frac{\lambda_{0}}{2 n_{s}} \frac{\partial\left(\arg \left(r_{1}\right)+\arg \left(r_{2}\right)\right)}{\partial \lambda} \frac{\lambda_{0}}{2 \pi} .
\end{aligned}
$$

$d_{\mathrm{eff}, i}$ gives rise to an effective cavity order $m_{C \text { eff }, i}, i=\lambda, \theta$

$$
m_{C \text { eff }, i}=\frac{d_{\mathrm{eff}, i}}{\lambda_{0} / 2 n_{s}} .
$$

When the resonances of the cavity are characterized by a high $F$, a rough estimate for $\eta_{\text {extr }}$ can be obtained: the Airy function can be approximated by a Dirac distribution for which $\eta_{\text {extr }}$ is translated to a ratio of discrete sums.

$$
\eta_{\text {extr }}=\frac{\sum_{i, \theta<\theta_{c}} \zeta_{i}}{\sum_{i} \zeta_{i}} .
$$

The numerator accounts for the extractable modes and the the denominator for all modes, both FP and guided modes. For the RCLED of Fig. 4(a), with the source located in the center of the cavity $\left(s_{1}\right)$, only even modes are excited with equal $\zeta_{i}$. With a single mode in the extraction cone (20) can be written as

$$
\eta_{\text {extr }} \cong 1 /\left(\left\lfloor m_{C \text { eff }, \theta}\right\rfloor / 2\right)
$$

The importance of $m_{C \text { eff }}$ for extraction efficiency estimation is thus obvious: the extraction efficiency depends on the penetration depth of the mirror. In general the penetration depth is positive, resulting in an increase of $m_{C \text { eff, } \theta}$. A novel design-the RC2LED - realizes a negative angular penetration depth using a nonperiodic high index-contrast mirror. As a result, coupling to small NA is enhanced (see Section VI) [39]. 


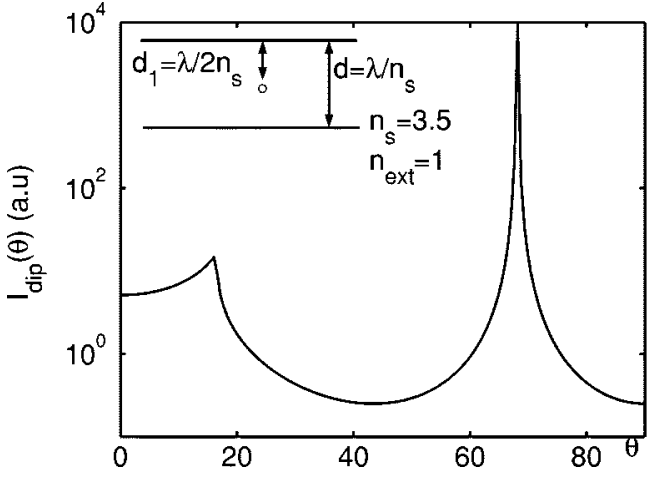

(a)

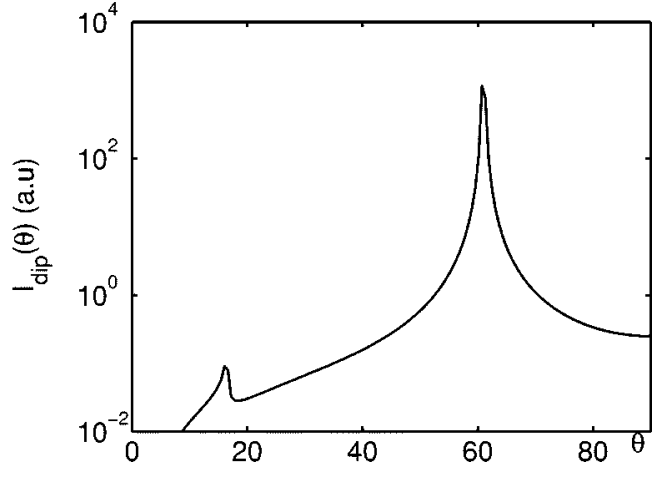

(b)

Fig. 5. Internal angle-resolved (a) TE intensity of horizontal dipole and (b) TM intensity of vertical dipole in a $\lambda$-cavity.

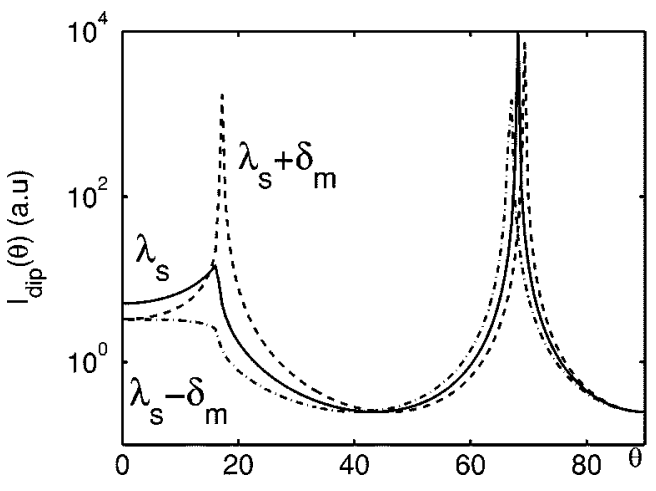

(a)

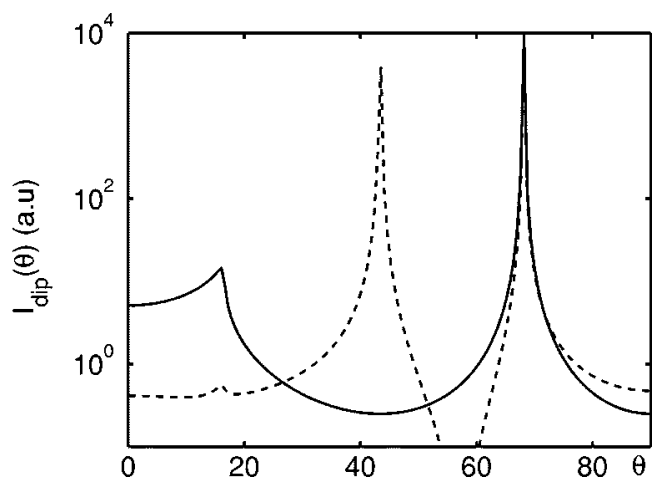

(b)

Fig. 6. (a) Internal angle-resolved TE intensity for $\lambda$-, $\left(\lambda+\delta_{m}\right)$-, $\left(\lambda-\delta_{m}\right)$-cavity $\left(\lambda_{s}=\lambda_{0} / n_{s}\right)$. (b) Internal angle-resolved TE intensity in a $\lambda$-cavity for dipole at antinode position of Fabry-Pérot mode (full line) and at node position of FP mode (dashed line).

\section{B. Dipole in Semiconductor Slab Cavity: Effect of Cavity Thickness and Position of Active Layer}

Fig. 5 shows a generic example of a monochromatic $\left(\lambda_{0}\right)$ horizontal dipole (TE) and vertical dipole (TM) in the middle of a semiconductor layer, thickness $d=\lambda_{s}$ and refractive index $n_{s}$ surrounded by air. This is a cavity with rather modest mirror reflectivity (about 30\%) for normal incidence and perfect reflectivity $(100 \%)$ for oblique incidence in the total internal reflection regime. Two resonances appear in the upwards emitted intensity through plane $z=0$ : a broad resonance from $\theta=0^{\circ}$ to $\theta=\theta_{c}$ corresponding with the extractable FP mode and a second high $Q$ resonance at $\theta=68^{\circ}$ for TE and $\theta=60^{\circ}$ for TM, or the guided mode trapped in the cavity. The FP mode shows a peak at $\theta=\theta_{c}$ although the phase resonance operation point, defined by (6) is fulfilled for $\theta=0$. This is due to the higher reflectivity coefficient of slanted angles, resulting in a stronger Airy factor than at phase resonance operation point. In a lossless cavity, the guided mode corresponds with a singularity in the power flux, as can be expected from (5). This singularity is not unphysical: integration over a finite solid angle results in a finite value.

To maximize $\eta_{\text {extr }}$, the thickness of the layer has to be optimized. The FP enhancement has to be located optimally in the extraction cone to maximize its extraction: $\eta_{\text {extr }}$ is proportional to the ratio of the area below the Airy function within the extraction cone to the total spanned surface. Fig. 4(b) shows that extraction will be maximal for the ideal cavity if the Airy-factor peak is located symmetrically in the $\left[\phi(0), \phi\left(\theta_{c}\right)\right]$ interval or $\phi\left(\theta_{0}\right)=\left(\phi(0)+\phi\left(\theta_{c}\right)\right) / 2$. With (6) and $\theta_{c} \simeq n_{\text {ext }} / n_{s}, \theta_{0}$ can be deduced and is approximately given by [40]

$$
\theta_{0} \simeq \frac{n_{\mathrm{ext}}}{\sqrt{2} n_{s}} .
$$

This skew propagation direction corresponds with an optimal cavity thickness correction $\delta_{m}$

$$
\delta_{m} \simeq \lambda_{0} \frac{n_{\mathrm{ext}}^{2}}{4 n_{s}^{2}} .
$$

With Snell's law $\left(n_{s} \sin \theta_{0}=n_{\text {ext }} \sin \theta_{0}^{\text {ext }}\right)$, this cavity thickness $d_{m}=\lambda_{s}+\delta_{m}$ results in an external angle of approximately $\theta_{0}^{\text {ext }}=45^{\circ}$. The detuning will influence the external emission profile strongly: the "rabbit-ears" in the emission pattern are an unavoidable property of highly efficient RCLEDs. This results in different design parameters for devices with an overall high efficiency and devices with a high efficiency toward a limited NA around the surface normal direction needed in optical fiber communication applications. The internal angle-resolved intensity is depicted in Fig. 6(a) for varying cavity thicknesses.

The position of the dipole in reference to the standing wave pattern defines the coupling strength of the source to this optical mode. It is expressed by the standing wave factor $\zeta\left(\phi_{2 \mathrm{eff}}\right)$. This factor reveals that the use of a thin active layer located at a standing wave node results in minimal coupling strength, while at an antinode height coupling strength is maximal. The optimal dipole position is thus at an antinode of the extractable FP mode as shown in Fig. 6(b), where the FP mode vanishes for 


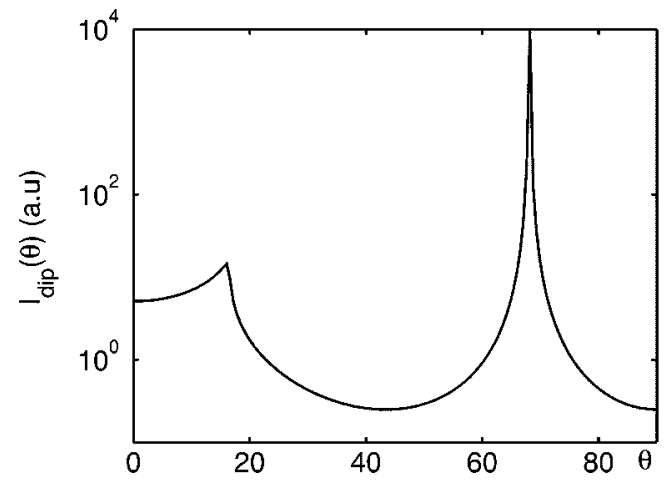

(a)

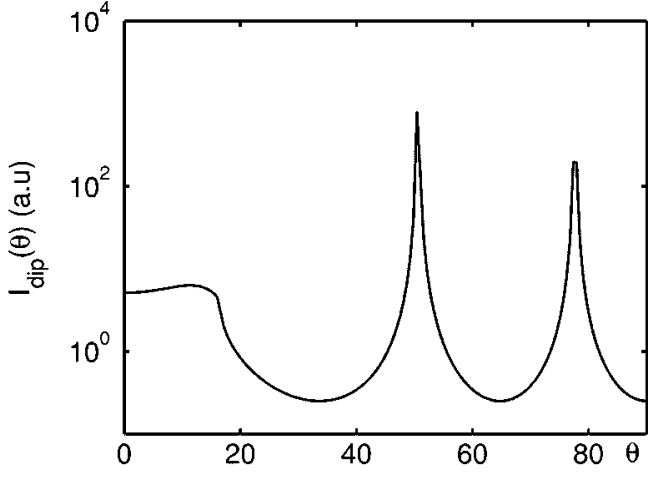

(b)

Fig. 7. Internal angle-resolved TE intensity for (a) $\lambda$-cavity and (b) $2 \lambda$-cavity.

a dipole at the level of a node. The interference effects are averaged out when a thick-at least $\lambda_{0} / 2 n_{s}$-bulk active layer is used, halving the overall enhancement as compared to the thin active layer placed at an antinode.

The effect of drastically increasing the cavity length is shown in Fig. 7 where $d$ is changed from $1 \lambda_{0} / n_{s}$ to $2 \lambda_{0} / n_{s}$, while the dipole is kept in the middle of the cavity. The intensity profile clearly shows a growing number of resonances when the cavity length is increased. Equation (21) can be applied to estimate the trend of $\eta_{\text {extr }}$ : increasing the reduced cavity length $m_{C \text { eff }}$ results in a drop of $\eta_{\text {extr }}$. This explains why an RCLED needs to have a small cavity length. Ideally, the total optical power is emitted in a single extractable FP mode, using a $\lambda / 2$-cavity. However, $\lambda$-cavities are in generally used in practical applications for several reasons. Due to losses, finite spectral width, etc., the coupling efficiency of the optical power to a single FP mode is not as efficient as Fig. 7 might suggest (see next section). Secondly, in case the cavity is set up by metallic mirror(s), a $\lambda / 2$ cavity implies that the distance of the active layer to the metal is very small and can result in considerable losses due to nonradiative energy transfer from the dipole to the absorptive metal [41]. For a perfect mirror with phase shift $\pi$ the distance is maximally $\lambda_{0} / 4 n_{s}$ (e.g., about $70 \mathrm{~nm}$ for a GaAs RCLED emitting at $980 \mathrm{~nm}$ ). For realistic metallic mirrors, with a phase shift different from $\pi$, this distance is even further reduced by some tens of nanometers (a reduction of about $45 \mathrm{~nm}$ for a GaAs RCLED emitting at $980 \mathrm{~nm}$ with Au-mirror). Finally, from a technological point of view, a $\lambda$-cavity is preferable to its thinner counterpart, and will in general be used in practical devices both when metallic mirrors or dielectric mirrors are used. A metallic mirror requires a heavily doped contact layer to ensure good electrical contact. Experiments evidenced that minimal thicknesses of several tens of nanometer $(50 \mathrm{~nm}$ for a GaAs RCLED emitting at $980 \mathrm{~nm}$ with Au-mirror [42]) are needed for the contact layer. As this contact layer (heavily doped GaAs) extends to a distance of some $20 \mathrm{~nm}$ from the active layer, there is not much space left for bandgap engineering to optimize the device. Multiple QW layers sandwiched in a carrier confinement structure [like graded refractive index (GRIN) confinement structures optimizing carrier caption [43]] typically have a total thickness of about $120 \mathrm{~nm}$. When dielectric mirrors are used, a sideways current supply is needed to pump the device. In this case again, a minimal thickness can be needed. To achieve proper carrier injection and low series resistance, extra layers can be added in the cavity.

\section{Cavity With DBR Mirror(s): Effect of Mirror Reflectivity}

Besides the cavity thickness tuning, the efficiency of the RCLED depends strongly on both amplitude and phase of the reflection coefficient of the cavity mirrors. Fig. 8 generically shows the amplitude and penetration depth of different mirrors as a function of the angle of incidence for TE and TM polarization: a semiconductor/metal interface, a semiconductor/air interface and a distributed Bragg reflector (DBR) mirror. A DBR mirror consists of a periodic quarter-wave stack of alternating high and low index material.

For both the back mirror and outcoupling mirror, the penetration depth has to be minimal to enhance the extraction efficiency $\eta_{\text {extr }} \sim 1 / m_{C \text { eff }}$ [see (21)]. For the outcoupling mirror, absorption losses have to be low.

The metallic mirror scores best for minimal penetration depth, but is preferably not used as an outcoupling mirror due to its high absorption losses. When metal is used as the back mirror, deposited on the semiconductor after epitaxial growth, the device will be bottom-emitting and a transparent substrate is needed, or a substrate removal. The metal will both serve as mirror and electrical contact.

The penetration depth of a DBR decreases with increasing refractive index-contrast. Approximate expressions for the penetration depth around the working point $\lambda_{0}=\lambda_{\mathrm{DBR}}$ and $\theta=0^{\circ}$, with $\lambda_{\mathrm{DBR}}$ the Bragg wavelength, are given in [44], for a large number $N$ of DBR pairs

$$
\begin{aligned}
d_{\text {pen }, \lambda} & \approx \frac{\lambda_{\mathrm{DBR}}}{4 n_{s}^{2}} \frac{n_{H} n_{L}}{n_{H}-n_{L}} \\
d_{\text {pen }, \theta} & \approx \frac{n_{s}^{2}}{2}\left(\frac{1}{n_{H}^{2}}+\frac{1}{n_{L}^{2}}\right) d_{\text {pen }, \lambda}
\end{aligned}
$$

with $n_{H}, n_{L}$, respectively, the highest and lowest refractive index of the DBR stack. The penetration depth decreases with decreasing number of DBR pairs. (24) and (25) define an upper limit for the respective penetration depths. It is important to note that $d_{\mathrm{pen}, \lambda}<d_{\mathrm{pen}, \theta}$ and this for all $N$. With $d_{\mathrm{pen}, \theta}$, the most significant parameter to determine $m_{C \text { eff }}$, the effect of high index-contrast mirrors on penetration depth will be less effective than might be expected from $d_{\text {pen }, \lambda}$, the commonly used definition for penetration depth. Not intuitive is the fact that $d_{\text {pen }, \theta}$, and hence, $m_{c \text { eff }, \theta}$ are minimal for $n_{L} \approx \sqrt{n}_{H}$. When a transparent material set with high refractive index-contrast 


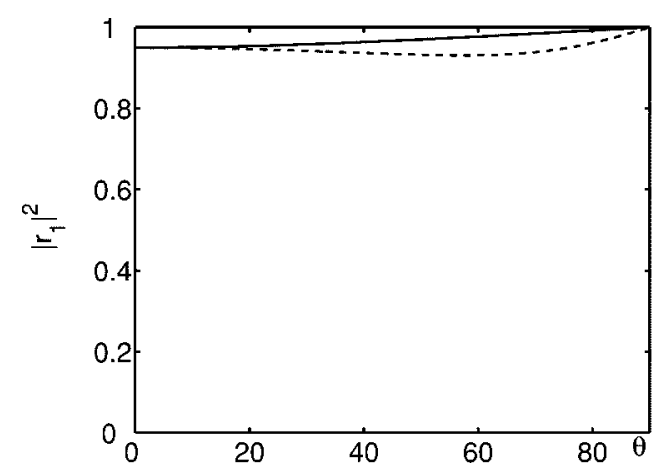

(a)

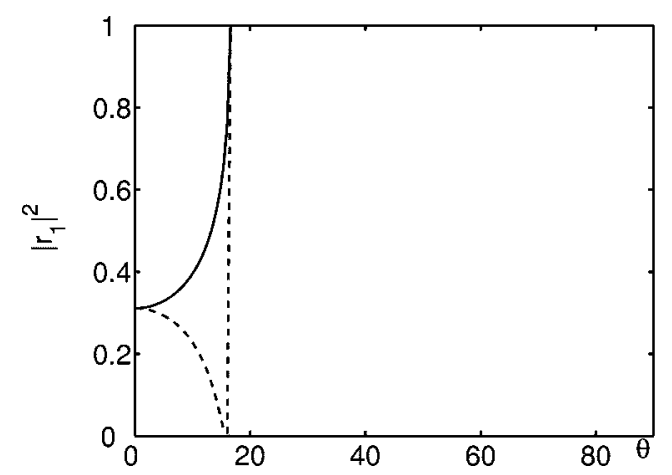

(c)

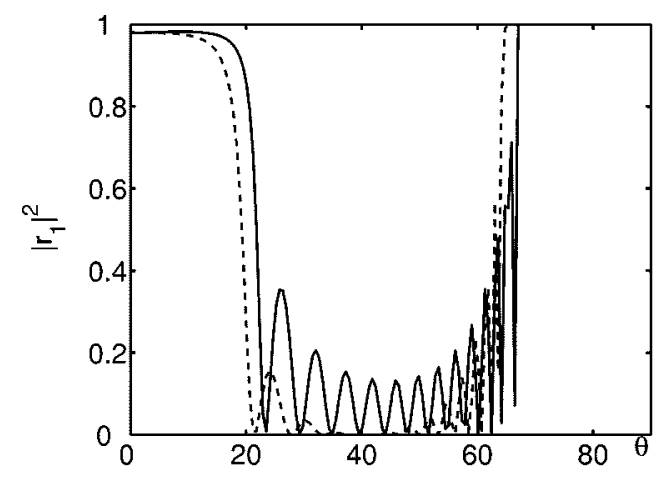

(e)

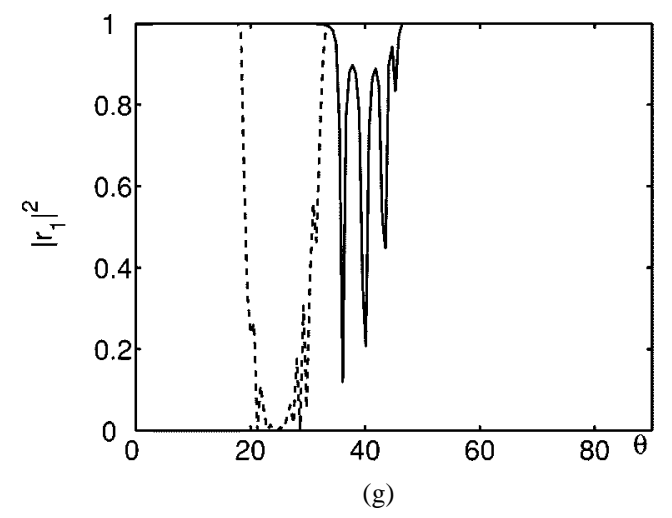

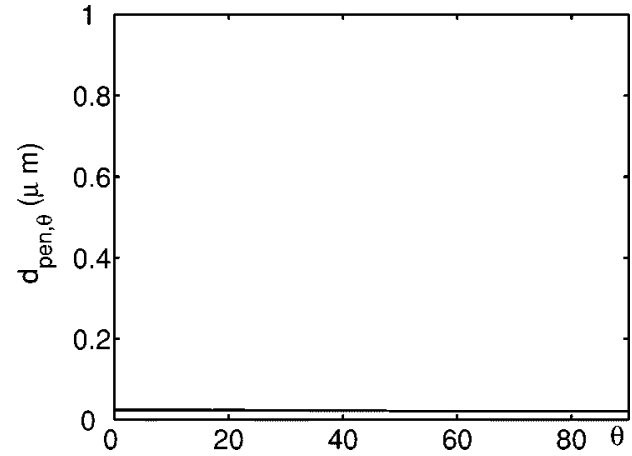

(b)

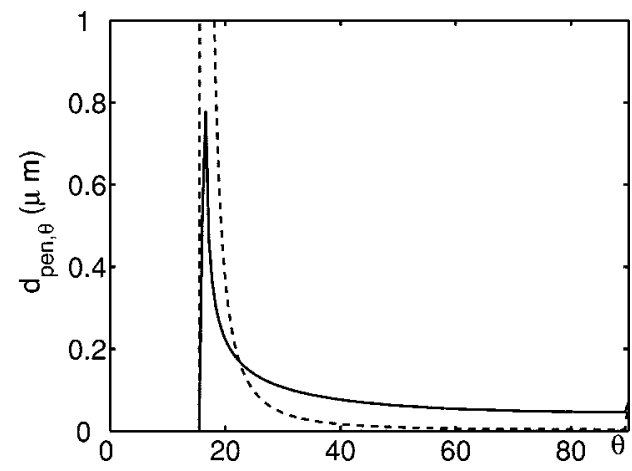

(d)

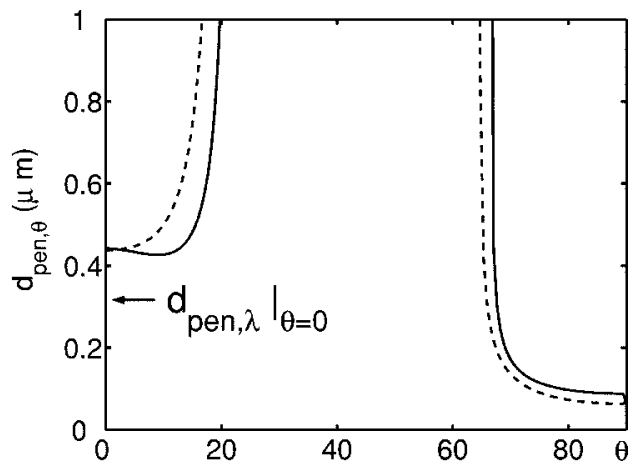

(f)

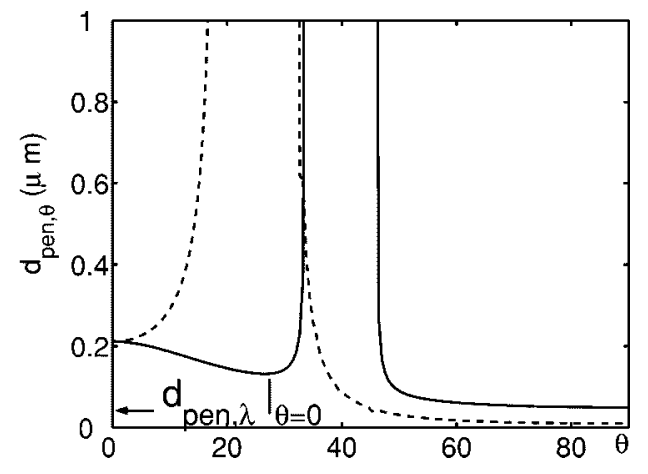

(h)

Fig. 8. Reflectivity and penetration depth as a function of the incident angle $\left(\lambda=\lambda_{\mathrm{DBR}}=0.98 \mu \mathrm{m}\right)$ Full line: TE-polarization. Dashed line: TM-polarization. (a)-(b) GaAs-Au interface. (c)-(d) GaAs-Air interface. (e)-(f) GaAs-(AlAs-GaAs DBR)-GaAs interface ( $N=30)$. (g)-(h) GaAs-(AlO $x$-GaAs DBR)-GaAs interface $(N=10)$.

can be found, a DBR is suitable for both back and outcoupling mirror.

In practical applications, a single facet extraction is preferred. This requires a very high reflectivity of the back mirror (ideally 1), which can be met by both a metallic mirror or a large stack
DBR. Optimizing light extraction at the output facet, requires moderate reflection coefficients and corresponding finesse $F_{\mathrm{FP}}$ and $Q_{\mathrm{FP}}$ for the FP mode. The reason is three-fold [27]: Considering a monochromatic source in a lossless cavity, $\eta_{\text {extr }}$ is proportional to the ratio of the area below the Airy function 


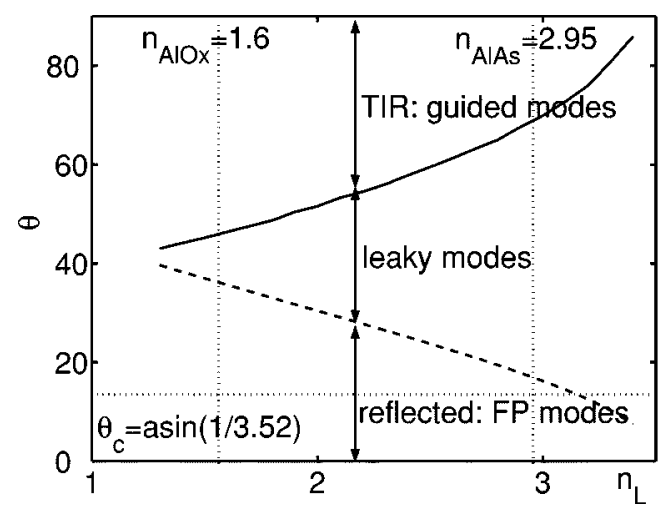

(a)

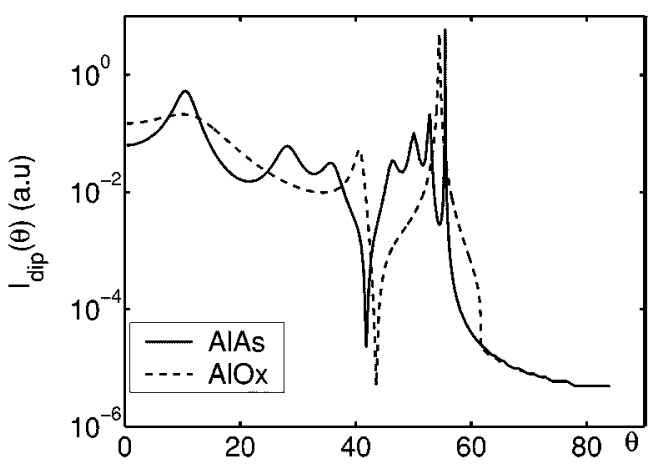

(b)

Fig. 9. (a) Angular limits of the reflection band and of TIR band as a function of the index-contrast $\left(n_{H}=3.52\right)$. (b) Internal angle-resolved TE intensity for $\lambda$-cavity with Au mirror and GaAs-AlAs and GaAs- $-\mathrm{AlO}_{x}$ DBR, respectively.

within the extraction cone to the total spanned surface [cf. (8) and (20)]. It is thus clear that the finesse of the FP mode has an optimal value, such that the peak resides well in the extraction cone, and above which value $\eta_{\text {extr }}$ increases only marginally. With the resonant peak given by (22), an approximate criterion can be deduced

$$
\begin{aligned}
\frac{1}{Q_{\mathrm{FP}}} & =\frac{\delta \phi}{\phi}=\delta \cos \theta<\frac{\left(1-\cos \theta_{c}\right)}{3} \\
& =\frac{1}{6} \frac{n_{\mathrm{ext}}^{2}}{n_{s}^{2}} \equiv \frac{1}{Q_{\mathrm{FP}}^{\text {crit }}}
\end{aligned}
$$

or $\left|r_{1}\right|>r_{1}^{\text {crit }}$, with $r_{1}^{\text {crit }}$ the reflection coefficient that gives rise to a $Q$ factor $Q_{\mathrm{FP}}^{\mathrm{crit}}$ as defined in (26).

Secondly, practical cavities have absorption losses. A moderate $\left|r_{1}\right|$ prevents a large number of round trips in the cavity, minimizing the absorption losses. If such losses are low enough, the FP resonance will not be affected, and criterion (26) will apply. In case of large losses, the width of the FP resonance remains large for increasing $\left|r_{1}\right|$, and maximum efficiency is reached when the "exit losses" or extraction equal the absorption losses, corresponding with some value $r_{1}^{\text {loss }}$. The criterion becomes: $\left|r_{1}\right|=\min \left(r_{1}^{\text {crit }}, r_{1}^{\text {loss }}\right)$.

Finally, due to the nonzero natural linewidth of the dipole source $\delta \lambda$ (the emitting material intrinsic spectrum), improving extraction efficiency at some wavelengths occurs at the expense of other wavelengths, which can cause a decrease of the spectrally integrated efficiency. If $Q_{\text {intr }}=\lambda / \delta \lambda=1 / \delta \cos \theta$ is lower than $3 /\left(1-\cos \theta_{c}\right)=Q_{\mathrm{FP}}^{\text {crit }}($ see $(26))$, it is of no use to enlarge the finesse of the cavity, that is $\left|r_{1}\right|>r_{1}^{\text {intr }}$, with $r_{1}^{\text {intr }}$ corresponding with $Q_{\text {intr. }}$. In semiconductors, the relative intrinsic spectral width tends roughly to scale with the wavelength due to thermal motion of carriers

$$
Q_{\mathrm{intr}}=\frac{\delta \lambda}{\lambda} \sim \lambda \sim k T / h \nu .
$$

Hence short wavelength RCLEDs [e.g., InGaP red RCLED (see Section V)] do not suffer from the linewidth penalty. In GaAs-based RCLEDs $\delta \lambda / \lambda$ is of the same order as $1 / 2 n^{2}$. In long wavelength RCLEDs, the broadening $\delta \lambda / \lambda$ is larger than $1 / 2 n^{2}$, which explains why it is very difficult to make 1.3 - or $1.55-\mu \mathrm{m}$ RCLEDs with high efficiency.
The rule of thumb for the optimal reflection of the outcoupling mirror can be summarized as

$$
\left|r_{1}\right|=\min \left(r_{1}^{\text {crit }}, r_{1}^{\text {loss }}, r_{1}^{\text {intr }}\right) .
$$

From Fig. 8, (21) and (28), it is clear that a DBR with a high refractive index contrast is a suitable outcoupling mirror. On the other hand, the angular response of a DBR (Fig. 8) gives evidence of a high reflectivity around $\theta=0^{\circ}$ [45]

$$
\Delta \cos \theta=\frac{\Delta \lambda}{\lambda_{\mathrm{DBR}}} \approx \frac{2}{\pi} \frac{\Delta n}{n_{s}}
$$

but vanishes for larger $\theta$. Reflection rises again when TIR inside the DBR is met. The part with low reflectivity gives rise to a continuum of optical modes, called the DBR-leaky modes. This is comparable with emission in bulk. These modes and the guided modes in the TIR regime are lost. The limits of the stop band and of TIR are drawn in Fig. 9(a) as a function of the low refractive index $n_{L}$ in a DBR stack when $n_{H}=3.52$. A high index-contrast material suppresses the leaky modes in favor of the extraction but most of all in favor of the guided mode [46]. The angle-resolved internal intensity of a metal/DBR cavity is depicted in Fig. 9(b) for the respective index-contrasts marked in (a).

In a microcavity bound by DBR-mirror(s), the Purcell-factor for horizontal dipole is generally close to unity (between 0.8 and 1.2 typically) except if the cavity thickness goes to 0 or $m_{c} \rightarrow 0$. The reason for this is two-fold. First of all, the DBR is leaky over a wide angular range which means that in this range the situation is little different from homogeneous material. Secondly, the penetration depth of the DBR leads to an enlarged effective cavity thickness, even with zero cavity thickness. This enlarges $m_{C \text { eff. }}$.

\section{Temperature Influence and Saturation}

Injection of carriers in the active region can be performed by optical pumping or current injection. For practical use, electrically pumped devices are far more preferred. This does not only call for an electrically optimized design next to an optically optimized design, generally requiring a compromise, but can lead to detrimental temperature effects. First, the higher the current density, the broader the intrinsic emission spectrum. This results 
in a decreased overlap between the cavity resonance and the intrinsic spectrum, and a decreased extraction efficiency. Secondly, as the current increases, the electrical dissipation in and around the active layer, caused by the nonradiative recombination and ohmic heating by the series resistance, results in a temperature increase of the active region. The temperature increase has two consequences. First, the internal quantum efficiency decreases (with $T_{0}$ a characteristic temperature) [30]:

$$
\eta_{\mathrm{int}}(T) \approx \eta_{\mathrm{int}, 0} \exp \left(-\frac{T}{T_{0}}\right)
$$

Second, the cavity resonance wavelength (mainly due to the temperature dependency of the refractive index, and little due to the thermal expansion of the material) and the intrinsic emission wavelength (due to the decreasing gap energy of semiconductors with increasing temperature) will shift at different rates toward longer wavelengths. The shift of the cavity resonance is much smaller than the shift of the peak wavelength emitted from the active semiconductor material. This results in a temperature dependent overlap between the intrinsic emission spectrum and the cavity enhancement, and a decreasing extraction efficiency when increasing current [47].

The decrease of efficiency as a function of the current level results in a saturation of the optical power. The decrease in efficiency at low currents is related to the increasing full-width at half-maximum (FWHM) of the intrinsic spontaneous emission profile. At higher current levels the thermal effects become more important, and the efficiency decreases faster. At a certain current level, the optical power does not increase anymore. This maximum power is proportional to the RCLED area. Typical values are in the order of several $\mu \mathrm{W} / \mu \mathrm{m}^{2}$, but do strongly depend on the thermal resistance of the ambient media.

If the current density of the device is known in advance, proactive design can partially intercept the temperature effect on efficiency at the working point. A detuning has to be chosen such that the overlap between intrinsic emission spectrum and the cavity enhancement increases as function of increasing temperature and is maximal at the operating temperature. The increase should at least compensate the decrease of the internal efficiency. This implies a decreased efficiency at low temperature compared to a cavity designed for maximum efficiency at the same temperature. The performance at the working point is best expressed as a differential efficiency (see Section IV).

\section{E. Large Diameter RCLEDs and Photon-Recycling}

The photons emitted in the guided wave can not escape the cavity and are-at first sight-lost. However, they can be reabsorbed in the active layer, and be re-emitted. This recycling effect increases the internal efficiency $\eta_{\text {int }}$, and thus, the overall external efficiency $\eta_{\text {ext }}$ of the device, as an electron will have a higher probability to produce a photon that escapes from the cavity. This is expressed by an "apparent internal quantum efficiency" $\eta_{\text {int }}^{\star}$

$$
\eta_{\text {int }}^{\star}=\eta_{\text {int }} \frac{1}{1-g \eta_{\text {int }}} .
$$

The reabsorption factor $g$ is defined as the ratio of the number of recycled photons to the total number of internally generated

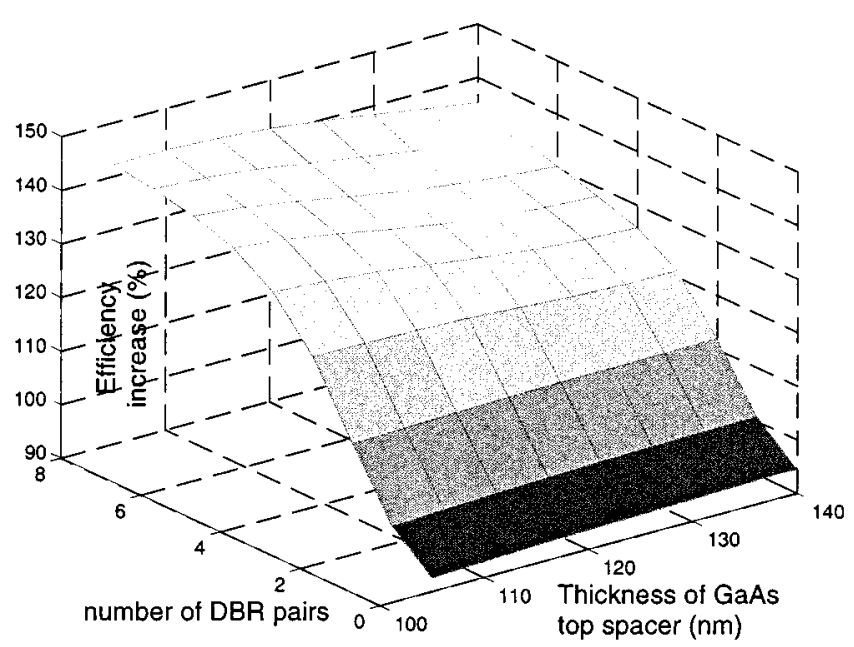

Fig. 10. Efficiency increase due to recycling as a function of top spacer thickness $d_{1}$ and number of GaAs-AlAs DBR pairs of GaAs $\lambda$-cavity emitting at $980 \mathrm{~nm}$.

photons. This ratio depends on two factors: the number of photons emitted into the guided mode and the number of guided photons that is reabsorbed in the active layer before they escape the cavity laterally or are absorbed in the rest of the cavity. The first depends on the layer structure (i.e., the cavity tuning). The second factor depends on the characteristic absorption length of the layer structure and the device diameter. The characteristic absorption length of a cavity is a function of the absorption coefficient of the active layer (which depends on the carrier density in the active layer) and the overlap between the internal field profile and the active layer. The guided mode is not necessarily concentrated around the active layer: the field profile can be concentrated in the DBR, resulting in a rather small overlap factor. A typical value of the absorption length is $50 \mu \mathrm{m}$. Consequently, the recycling effect is present in large diameter devices and negligible (or even absent) in small diameter devices. Fig. 10 shows the calculated increase of $\eta_{\text {int }}$ in case of full lateral reabsorption (i.e., device diameter much larger than reabsorption length) and assuming an internal quantum efficiency $\eta_{\text {int }}=90 \%$. The recycling increases as function of the number of DBR pairs, as this results in a more concentrated field profile around the active layer, and thus, an increased overlap. The influence of a variation of the thickness of the top spacer $d_{1}$ of the cavity is less pronounced. In large devices, the recycling effect results in a 1.4 times enhancement of the overall efficiency.

\section{F. Modulation Bandwidth of RCLEDs}

Besides efficiency, the modulation bandwidth is of uttermost importance in communication applications [15], [47]. The response of an LED can be described by rate equations

$$
\begin{aligned}
\frac{d n}{d t} & =\frac{I(t)}{q V_{\text {act }}}-\frac{n}{\tau_{\text {nrad }}}-B n^{2} \\
P & =\hbar \omega \eta_{\text {extr }} B n^{2} V_{\text {act }}
\end{aligned}
$$

with $I(t)$ the injected current, $V_{\text {act }}$ the volume of the active region, $\tau_{\text {nrad }}$ the nonradiative lifetime and $n$ the carrier concentration. $B$ is the bimolecular recombination coefficient, describing 
the radiative recombination speed. For simplicity, the Auger recombination, dominant at very high carrier concentrations is neglected (only in long wavelength devices, Auger recombination can be an important effect, and should not be neglected) [48].

The radiative recombination speed is found after averaging the recombination lifetime of an electron-hole pair in the semiconductor over all available energy levels of the electrons and holes in the active region. Therefore, it includes the Purcell effect. This implies that the microcavity effect which alters the optical mode density and thus the Purcell factor, will influence the dynamics of the RCLED. However, as mentioned in Section II.B, the spontaneous emission rate enhancement depends on the emission wavelength, the cavity parameters and the dipole orientation. The change in lifetime is limited for practical cavities with an AlAs-GaAs DBR [38]. Several experiments have been reported [7], [49]. The largest decrease in recombination lifetime is obtained with a high- $Q$ cavity (corresponding to a small cavity bandwidth), optically pumped RCLEDs and an overtuned cavity. The measured decay time was $10 \%$ shorter compared to standard LEDs.

Besides the effect of the cavity on the bimolecular recombination coefficient, the speed behavior of RCLEDs is similar to standard LEDs. Although the radiative recombination lifetime is slow, compared to stimulated emission, special enhancements can be used to speed up the devices. Examples are the use of a background doping, the use of peaking current driver circuits or voltage drivers, and the use of smaller active regions (such as QWs). Photon recycling (see Section III.E), based on absorption and re-emission of emitted photons affects the bandwidth of the (RC)LED. It increases the LED's response time to an electrical input signal. Photon recycling can be beneficial for high-efficiency applications but undesirable for high-speed communication devices.

\section{GUIDELINES FOR COMPARISON OF HIGH EFFICIENCY LEDS}

There are many ways to compare the performance of high-efficiency LEDs and the preferred technique depends strongly on the application. In spite of the importance of the external efficiency $\eta_{\text {ext }}$, it has to be emphasized that an objective comparison of the different techniques to enhance $\eta_{\text {ext }}$ can only be obtained when a complete analysis is done. This includes in the first place a clear indication of the definition of the indicated efficiencies and saturation values and a comparative analysis of some basic aspects. A checklist is given hereafter.

The external performance of an LED can be expressed in different ways, making comparison of devices to be done with care. The commonly used definitions to express the efficiency and saturation values are presented in Table II.

The external quantum efficiency $\eta_{\text {ext }}$ is the most commonly used definition expressing the number of photons per injected electron. Either the overall $\eta_{\text {ext }}$ can be given, or the $\eta_{\text {ext }}$ to a limited numerical aperture (NA). The corresponding relevant absolute value is the maximum photon flux $S_{\text {phot }}^{\mathrm{sat}}$. These definitions allow to compare LEDs with a different emission wavelength.

The wall plug efficiency $\eta_{\mathrm{wp}}$ is an alternative for $\eta_{\text {ext }}$. It takes into account the series resistance of the diode, as it is defined as the ratio of the emitted optical power to supplied electrical
TABLE II

COMMONLY USED DEFINITIONS TO EXPRESS EFFICIENCY AND SATURATION VALUES

\begin{tabular}{|c|c|}
\hline Efficiency & Saturation value \\
\hline$\eta_{e x t}=\frac{P_{o p t} / \hbar \omega}{I / q}$ & $\begin{array}{c}S_{\text {phot }}^{\text {sat }}=\frac{P_{o p t}^{\max }}{\hbar \omega} \\
{\left[s^{-1}\right]}\end{array}$ \\
\hline$\eta_{w p}=\frac{P_{o p t}}{I V}$ & $\begin{array}{c}P^{s a t}=P_{o p t}^{\max } \\
{[W]}\end{array}$ \\
\hline $\begin{aligned} \eta_{p h o t}= & 683 V_{C I E}(\lambda) \eta_{w p} \\
& {[\mathrm{~lm} / W] }\end{aligned}$ & $\begin{array}{c}L^{s a t}=683 V_{C I E}(\lambda) P^{s a t} \\
{[l m]}\end{array}$ \\
\hline$\eta_{\text {dens }}=\frac{1 / A_{\text {extr }}}{1 / A_{\text {act }}}$ & $\begin{array}{r}M^{s a t}=\frac{P_{o p t}^{\max }}{A_{\text {extr }}} \\
{\left[W / m^{2}\right]}\end{array}$ \\
\hline $\begin{array}{c}\eta_{r d n}=\frac{d P_{o p t} / d \Omega A_{e x t r}}{I V} \\
{\left[W / W \cdot m^{2} \cdot s r\right]}\end{array}$ & $\begin{array}{r}R^{s a t}=\frac{d P_{o p t}^{\max }}{d \Omega A_{\text {extr }}} \\
{\left[W / m^{2} . s r\right]}\end{array}$ \\
\hline $\begin{array}{c}\eta_{l u m}=683 V_{C I E}(\lambda) \eta_{r d n} \\
{\left[c d / W \cdot m^{2}\right]}\end{array}$ & $\begin{array}{c}B^{s a t}=683 V_{C I E}(\lambda) \frac{d P_{o p t}^{\max }}{d \Omega A_{e x t r}} \\
{\left[c d / m^{2}\right]}\end{array}$ \\
\hline
\end{tabular}

power $I V$, with $V$ the bias voltage $(\mathrm{V})$. Again, an NA can be specified. The maximal output power $P^{\text {sat }}$ indicates the saturated power or radiant flux.

The photometric efficiency $\eta_{\text {phot }}$ weighs the wall plug efficiency with the relative luminosity $V_{\mathrm{CIE}}(\mathrm{lm} / \mathrm{W})$, defined by the Commission Internationale de l'Eclairage (CIE). $V_{\mathrm{CIE}}$ is proportional to the effectiveness in stimulating the human visual sense. $\eta_{\text {phot }}$ is, thus, only defined for LEDs emitting visible light. The maximal luminous flux $L^{\text {sat }}$ scales the radiant flux with the relative luminosity.

The density efficiency $\eta_{\text {dens }}$ is a measure for the efficient active use of the available surface. It is defined as the ratio of the inverse of the extraction surface $A_{\text {extr }}$ to the inverse of the surface of the active layer $A_{\text {act }}$. In a RCLED $A_{\text {act }} \approx A_{\text {extr }}$ and thus very high. This parameter is important for scaling of high brightness devices. $M^{\text {sat }}$ expresses the maximal total near-field power density or radiant emittance.

The radiant efficiency $\eta_{\mathrm{rdn}}$ defines the conversion efficiency of the electrical power to the peak radiance. $R^{\text {sat }}$ is the saturation radiance. The radiance is a quantity that-at best-is conserved in an imaging system: the radiance of the image cannot be higher than that of the object. Therefore, high radiance is important in any application where light needs to be focused to a small spot or where light needs to be coupled into a fiber with finite core size and numerical aperture. While the radiance of a RCLED is still orders of magnitude lower than that of a laser (for a given power), it is easily orders of magnitude larger than that of an LED chip with light extraction from all chip sides or with small density efficiency.

The luminous efficiency $\eta_{\text {lum }}$ weighs the radiant efficiency with the relative luminosity $V_{\mathrm{CIE}}$. Brightness is defined as the optical luminous power per unit solid angle $(\Omega)$ per unit surface 
TABLE III

REFRACTIVE INDEX CONTRAST IN COMMONLY USED MATERIAL SYSTEMS

\begin{tabular}{c||c|c|c}
\hline$\lambda$ & high index mat. & low index mat. & $\Delta \mathrm{n}$ \\
\hline \hline $0.65 \mu \mathrm{m}$ & $\begin{array}{c}\mathrm{Al}_{0.5} \mathrm{Ga}_{0.5} \mathrm{As} \\
\mathrm{n}=3.47\end{array}$ & $\begin{array}{c}\mathrm{Al}_{0.95} \mathrm{Ga}_{0.05} \mathrm{As} \\
\mathrm{n}=3.14\end{array}$ & 0.33 \\
\hline $0.85 \mu \mathrm{m}$ & $\begin{array}{c}\mathrm{Al}_{0.15} \mathrm{Ga}_{0.85} \mathrm{As} \\
\mathrm{n}=3.5\end{array}$ & $\begin{array}{c}\mathrm{AlAs} \\
\mathrm{n}=2.98\end{array}$ & 0.52 \\
\hline $0.98 \mu \mathrm{m}$ & $\begin{array}{c}\mathrm{GaAs} \\
\mathrm{n}=3.52\end{array}$ & $\begin{array}{c}\mathrm{AlAs} \\
\mathrm{n}=2.95\end{array}$ & 0.57 \\
\hline $0.98 \mu \mathrm{m}$ & $\begin{array}{c}\mathrm{GaAs} \\
\mathrm{n}=3.52\end{array}$ & $\begin{array}{c}\mathrm{Al0x} \\
\mathrm{n}=1.6\end{array}$ & 1.92 \\
\hline $1.3 \mu \mathrm{m}$ & $\begin{array}{c}\text { InGa } 0.23 \mathrm{As}_{0.50} \mathrm{P} \\
\mathrm{n}=3.42\end{array}$ & $\begin{array}{c}\mathrm{InP} \\
\mathrm{n}=3.21\end{array}$ & 0.21 \\
\hline $1.55 \mu \mathrm{m}$ & $\begin{array}{c}\text { InGa } 0.38 \mathrm{As}_{0.82} \mathrm{P} \\
\mathrm{n}=3.47\end{array}$ & $\begin{array}{c}\text { InP } \\
\mathrm{n}=3.17\end{array}$ & 0.3 \\
& \multicolumn{3}{|}{}
\end{tabular}

(A). $\eta_{\text {lum }}$ and $B^{\text {sat }}$ are only defined for LEDs emitting visible light, due to the relative luminosity in their definition. Unlike the efficiency, the power and brightness of an LED can be augmented when the pumping level is increased. The commercial labeling "high brightness" LEDs is strongly linked with "high efficiency" as the saturation level of a device at high pumping levels can only be increased when efficiency is increased.

A distinction has to be made between an efficiency and a differential efficiency. The differential efficiency defined as a power increase due to an infinitesimal rise of the current, will typically be higher than the absolute efficiency. The reason can be excessive nonradiative recombination, parallel current paths, saturation effects,....

- Is the device optically or electrically pumped? Electrically pumped devices demand electrical contacts, complex doping profile, etc. This tends to go together with geometrical issues that are not always favorable for $\eta_{\text {ext }}$.

- What is the emission wavelength? The intrinsic linewidth penalty, (27), scales with the wavelength. Moreover, the wavelength more or less defines the used material system. Table III summarizes commonly used material systems for different wavelength ranges. The refractive index contrast that can be realized varies strongly from wavelength range to wavelength range. As mentioned in Section III, the refractive index contrast is an important figure to determine the penetration depth of the DBR, and thus, the extraction efficiency.

- $\eta_{\text {extr }}$ scales roughly spoken with $\left(1-\cos \left(\theta_{c}\right)\right) \approx 1 / 2 n_{s}^{2}$. This has to be kept in mind when comparing the performance of RCLEDs with different refractive index $n_{s}$ e.g., polymer RCLEDs and semiconductor RCLEDs.

- Is the device encapsulated by an epoxy dome? Embedding the semiconductor device in a transparent plastic with a dome shape, enlarges the extraction cone in the semiconductor, and hence, its $\eta_{\text {extr. }}$. The waves impinge more or less normally on the surface of the large dome and are extracted.

- Is the device emitting through a single surface or do several surfaces contribute to the extraction? Some applications, e.g., fiber coupling and array devices do demand a single surface emitting diode. Is the diameter specified?

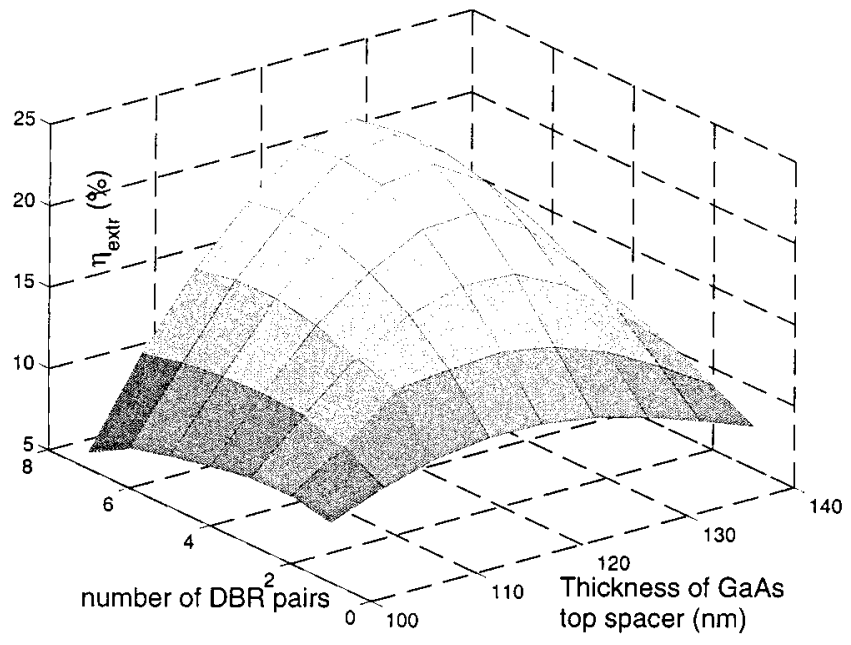

Fig. 11. Simulation of $\eta_{\text {cxtr }}$ of a 980-nm RCLED as a function of top spacer thickness $d_{1}$ and number of DBR pairs.

- The simplicity of fabrication: How easy can the production process be adopted for mass production?

- Dependent on the application, advanced characteristics-directivity, speed-can play an important role in the suitability of the device.

\section{STATE OF THE ART}

An overview of high-efficiency RCLEDs presented in literature is given. The overview is limited to electrically injected devices without epoxy encapsulation, unless explicitly mentioned.

\section{A. $980 \mathrm{~nm}$}

Unlike the 650 and $850-880 \mathrm{~nm}$ RCLEDs (cf. below), there is up until now, no killer application for 980-nm infrared devices. Moreover, there is a strong competition of 980-nm VCSELs. Nevertheless, thorough investigation on these devices has been carried out and is still going on. The GaAs- $\mathrm{Al}(\mathrm{Ga}) \mathrm{As}$ material system is used with InGaAs high-quality strained QWs for active material. Contrary to $650 / 850-880 \mathrm{~nm}, \mathrm{GaAs}$ is transparent for $980-\mathrm{nm}$ light. This makes devices emitting at $980 \mathrm{~nm}$ preferable for proof of principle experiments. Highly efficient bottomemitting devices, grown by MOCVD are reported in [17]. With a Au-(GaAs-AlAs)-DBR asymmetric $\lambda$-cavity, the metal layer both serving as electrical contact and as mirror, and three InGaAs-strained QWs, the overall external efficiency of $80-\mu \mathrm{m}$ devices is up to $17 \%$. When the diameter is larger, photon-recycling is more significant. An overall external efficiency up to $23 \%$ is obtained for large diameter $( \pm 1 \mathrm{~mm})$ RCLEDs, or a 1.4 enhancement. The sensitivity of the $\eta_{\text {extr }}$ to the cavity tuning and the influence of the reflectivity of the bottom DBR, expressed in number DBR pairs, is shown in Fig. 11. A deviation of the cavity thickness in the order of $\lambda_{s} / 10$ from the optimal value can result in a reduction of the efficiency by a factor three. The performance of the RCLED is in direct relation with the precision of the growth of the cavity thickness. This also accounts for devices in other wavelength ranges.

The measured eye diagram of a voltage-driven 980-nm RCLED (diameter is $30 \mu \mathrm{m}$ ) is shown in Fig. 12. Subnanosecond 


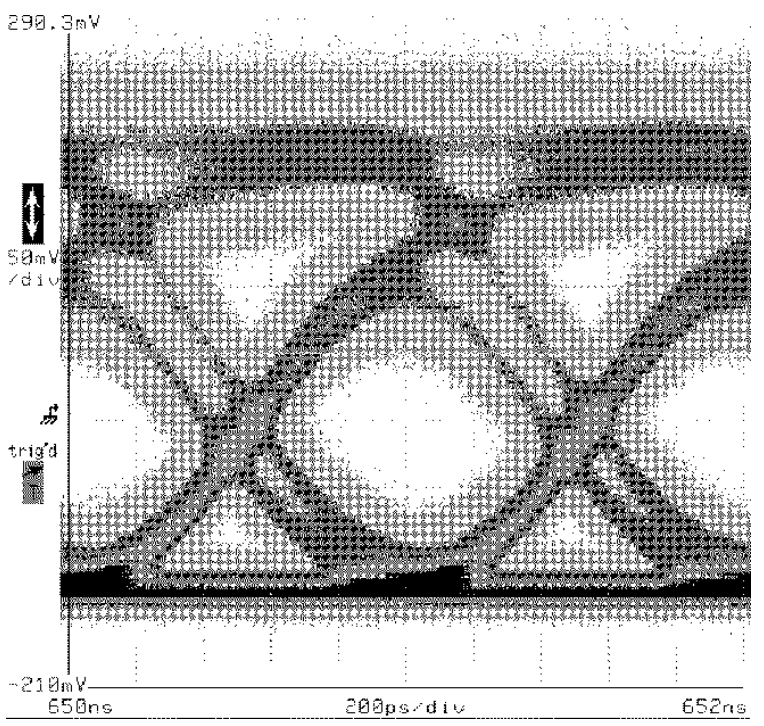

Fig. 12. Measured eye diagram of voltage-driven 980-nm RCLED at $1 \mathrm{~Gb} / \mathrm{s}$ $($ diameter $=30 \mu \mathrm{m})$

rise and fall times of the optical signals and communication with open eye diagrams at over $1 \mathrm{~Gb} / \mathrm{s}$ have been achieved [50]. The structure and doping profile are the same as the above-mentioned high-efficiency devices, but due to the smaller diameter, the overall external efficiency drops to $11 \%$.

Leaky DBR modes, metal mirror absorption and a trapped guided mode are the main loss channels. According to Section III, a higher index-contrast DBR could result in higher efficiencies due to the suppressed leaky modes and decreased penetration depth $\left(d_{\text {pen, }, \theta}\right)$. It can be achieved by laterally oxidizing the $\mathrm{Al}(\mathrm{Ga})$ As layers to obtain a high index-contrast $\mathrm{AlO}_{x}-\mathrm{GaAs}$ DBR mirror $\left(n_{\mathrm{AlO}_{x}} \approx 1.6\right)$. There are some drawbacks however. This electrically isolating material necessitates advanced techniques like intracavity contacts. Moreover, the use of a high contrast DBR is less appreciated in combination with a metallic top mirror: for a high contrast DBR, the reflectivity of even a single pair is comparable with the gold mirror, resulting in detrimental metal absorption losses [cf. (28)]. The benefits of the use of a high index-contrast $\mathrm{AlO}_{x}-\mathrm{GaAs}$ DBR are, thus limited to top-emitting devices not using a metallic mirror. A top-emitting device, aperture diameter $11 \mu \mathrm{m}$, with a lower 6.5 $\mathrm{AlO}_{x}-\mathrm{GaAs}$ DBRs and a one-two period upper $\mathrm{SiO}_{2}-\mathrm{ZnSe}$ DBR on the $\lambda$-cavity, and a tunnel contact junction to confine the laterally injected current under the DBR shows external differential quantum efficiencies as high as $27 \%$ [19]. A laterally injected top emitting $\lambda$-cavity with a single 3.5 pair AlOx-GaAs bottom DBR, diameter $350 \mu \mathrm{m}$, shows $\eta_{\text {ext }}$ as high as $27 \%$, encapsulated $28 \%$ [46].

\section{B. $650 \mathrm{~nm}$}

650-nm emitting devices are commercially important for plastic optical fiber (POF) based communication. Due to their visible wavelength, they can also serve a broad range of noncommunication applications for which a high efficiency is important. Like in standard LEDs, the GaAsP material system is increasingly substituted by the high-quality AlGaInP. Due to the absorbing substrate ( $\mathrm{GaAs}$ or $\mathrm{Ge}$ ), the device is preferably top emitting with a cavity sandwiched in between two DBR mirrors and an appropriate current injection design. Devices in the 600- to 650-nm range are described by several research groups, showing $\eta_{\text {ext }}$ ranging from some percents to 7\% [51]-[54]. A highly efficient top-emitting RCLED grown by MOCVD operating at $650 \mathrm{~nm}$ and having a low forward voltage is reported in [55]. A $300 \mu \mathrm{m} \times 300 \mu \mathrm{m}$ encapsulated device shows a $\eta_{\mathrm{wp}} \approx 10.2 \%$. The device, a $\lambda$-cavity with GaInP active layer enclosed by $2 \mathrm{Al}_{x} \mathrm{Ga}_{1-x} \mathrm{As}$ DBRs, is ready for large scale production.

\section{C. $850-880 \mathrm{~nm}$}

Apart from (Fast)-Ethernet LAN data links, the target applications of 850-880 $\mathrm{nm}$ devices are remote control and infrared communication as regulated by Infrared Data Association (IrDA) (mainly because of the availability of low-cost $\mathrm{Si}$-based detectors). The demands for these applications are different from telecom applications. While brightness and bandwidth are less important, absolute power, overall efficiency, and low cost are of paramount importance. The obvious material system for this wavelength range is GaAs, as its bandgap corresponds with this wavelength range. On the other hand, this implies that the use of GaAs in the substrate, as part of the DBR, etc. will absorb this light, and thus, its use has to be minimized. An 850-nm top-emitting device, consisting of a $\lambda$-cavity sandwiched between two DBRs grown by MOCVD, shows an overall efficiency $\eta_{\text {ext }}$ of $14.6 \%$ [56]. The top mirror is a 1.5 pair $\mathrm{Al}_{0.15} \mathrm{Ga}_{0.85} \mathrm{As}-\mathrm{AlAs} \mathrm{DBR}$, the bottom mirror a 20 pairs DBR. The decreased refractive index-contrast compared with a GaAs-AlAs DBR results in the need of a thicker DBR-stack. Current injection was optimized with a selectively oxidized current window with a diameter of $180 \mu \mathrm{m}$.

A 880-nm monolithic top-emitting device with $\lambda$-cavity, a 20 pairs $n$-doped $\mathrm{Al}_{0.2} \mathrm{Ga}_{0.8} \mathrm{As}-\mathrm{Al}_{0.9} \mathrm{Ga}_{0.1}$ As bottom $\mathrm{DBR}$ and a five-seven pairs top DBR, all grown by solid-source MBE is reported in [57]. With an emission window of $80 \mu \mathrm{m}$ and an epoxy cap, $\eta_{\text {ext }}$ is $16 \%$.

\section{D. $1300-1550 \mathrm{~nm}$}

The principal material system for the pre-eminently telecomwavelength devices is InP. In addition to the broader intrinsic spectrum of long wavelength devices [see (27)], the restrictions of the appropriate material systems (cf. long wavelength VCSELs), limits the maximal efficiency of long wavelength devices. The problem lies in the low refractive index-contrast that can be realized with InP lattice-matched alloys to form the DBR, resulting in large penetration depths. Highly efficient 1300-nm large diameter devices ( $2 \mathrm{~mm}$ ) with a peak quantum efficiency of $9 \%$ are reported in [58] using a monolithic cavity grown by MOCVD. The electrically pumped device is bottom emitting, using an asymmetric Au/DBR InP $\lambda$-cavity with three 4.5-nm $\mathrm{InGa}_{0.12} \mathrm{As}_{0.56} \mathrm{P}$ strained QWs. The low refractive index contrast of the 5.5 pair $\operatorname{InGa}_{0.23} \mathrm{As}_{0.50} \mathrm{P}-\mathrm{InP} \mathrm{DBR}$ is the main drawback.

Research toward high quality epitaxial DBR mirrors is driven by VCSEL research. Contrary to RCLEDs, the need for high index-contrast mirrors in VCSEL's arises from the sole need for highly reflective mirrors, whatever their penetration length, say $R>99 \%$. A low $\Delta n$ requires a large number of DBR mirror 


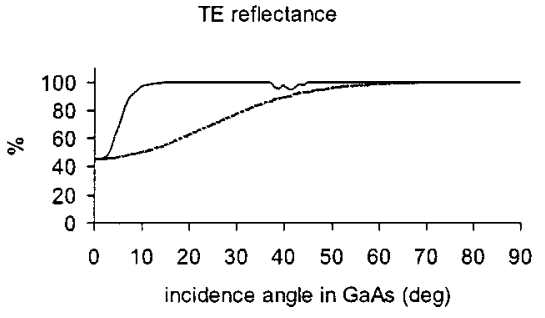

(a)

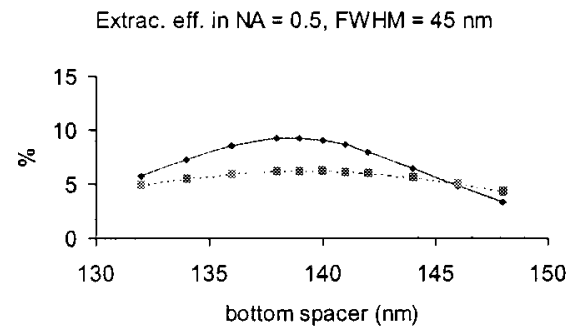

(c)

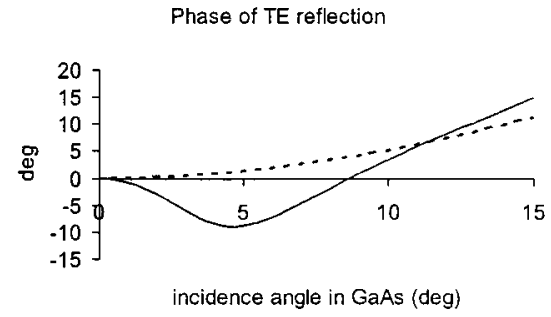

(b)

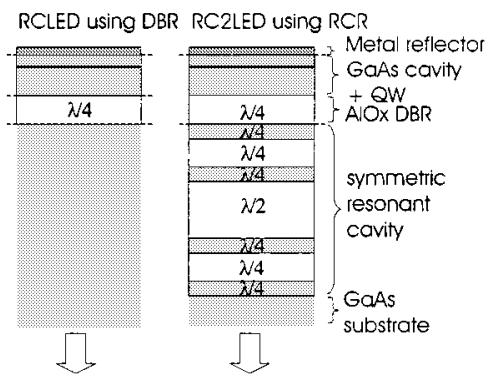

(d)

Fig. 13. RC2LED. (a) TE reflectance for DBR and RCR, as seen from the GaAs cavity (Full line: RCR. Dashed line: DBR). (b) Phase of TE reflection for DBR and RCR, as seen from the GaAs cavity (Full line: RCR. Dashed line: DBR). (c) Simulated extraction efficiency into NA = 0.5 (Full line: RCR. Dashed line: DBR). (d) RC2LED.

pairs in order the achieve the high reflectivity, translated into several growth-related problems. Other InP-lattice matched alloys are reported in VCSEL literature for fabrication of high refractive index-contrast DBR mirrors: especially aluminum and antimonide containing compound semiconductors are promising candidates [59].

On the other hand, VCSEL research has an increasing interest in the development of GaAs-based devices for long wavelength applications, i.e., active material compatible with GaAs. Next to the possibility of high index-contrast GaAs-AlAs Bragg mirror, this system is more temperature insensitive and cheaper. A path not yet entered by RCLEDs, is the use of GaInNAs as active material [60] to emit at $1300 \mathrm{~nm}$. The use of quantum dots (QD) has been investigated in several research groups. With self-assembled InAs-InGaAs QDs emitting at $1300 \mathrm{~nm}$ in a single mirror $\mathrm{Au}$ ) cavity, grown by solid-source MBE on a GaAs substrate, an external quantum efficiency of $1 \%$ at room temperature is obtained, limited by the low radiative efficiency of $13 \%$ of the QDs.

An MOCVD InP-based RCLED of diameter $80 \mu \mathrm{m}$ emitting at $1550 \mathrm{~nm}$ with a $6.8 \%$ external quantum efficiency is cited in [62]. The device is bottom emitting, using an asymmetric $\mathrm{Au} / \mathrm{DBR}\left(12\right.$ pairs $\mathrm{InGa}_{0.38} \mathrm{As}_{0.82} \mathrm{P}-\mathrm{InP}$ ) cavity. The active region consists of three $7.5 \mathrm{~nm} \mathrm{In} \mathrm{In}_{0.84} \mathrm{Ga}_{0.16} \mathrm{As}_{0.74} \mathrm{P}_{0.26} \mathrm{QWs}$. An electrically pumped top-emitting all epitaxial InGaAsP-InP DBR/DBR RCLED is reported in [63], optimized for high coupling efficiency to fiber, the main demand for this wavelength, instead of overall efficiency.

\section{AdVANCED TECHNIQUES}

\section{A. RC2LED}

In the RC2LED, a symmetric resonant cavity is added to the outcoupling mirror [39]. This novel mirror design, the resonant cavity reflector (RCR) yields high reflectance for off-axis incidence, combined with a moderate reflectance for normal incidence. This results in a narrower radiation pattern. Also, because the angular penetration depth is negative, extra resonances are created within the extraction cone, which boosts the extraction efficiency. These TE reflection properties of the RCR are depicted in Fig. 13(a)-(b) and compared with the corresponding DBR, sketched in (d). These factors combine to yield an extraction efficiency to a given NA which can be $50 \%$ to $100 \%$ higher than conventional RCLEDs. The extraction efficiency in a NA $=0.5$, for a GaAs-AlOx RC2LED (see [39]) with Gaussian spectrum with FWHM $=45 \mathrm{~nm}$ is shown in Fig. 13(c). This idea has not been verified experimentally yet.

\section{B. Photonic Crystal Assisted RCLED}

The main drawback of planar RCLEDs is the distribution of the optical power over several optical modes, while only a fraction will be extracted. Leaky modes and guided modes are lost, except through partial photon-recycling by reabsorption (see Section III.E). When the planarity of the RCLED is abandoned, control on the inplane dimensions can be obtained. The world of photonic crystals (PCs) and resonant cavities meet in the photonic crystal assisted RCLED (PCA-RCLED). A PC is a one-, two-, or three-dimensional (1-D, 2-D, 3-D) periodic corrugated medium. Its wavelength-scale period and high refractive index contrast can strongly influence the optical mode density. Apart from the 1-D PC, in the form of a DBR mirror already present in most RCLEDs, a 2-D PC can be added in the plane of the device. Different top-emitting configurations are sketched in Fig. 14. Configuration (a) and (b) inhibit emission in the guided mode, (c) and (d) extract the guided mode using a 2-D PC.

1) Inhibition of Guided Mode: The periodic corrugation can provide a bandgap in the dispersion relation of the guided modes at the emission frequencies. Emission will then initially be pre- 


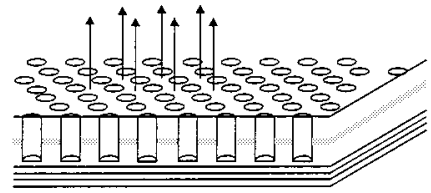

(a)

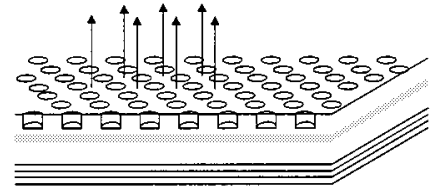

(c)

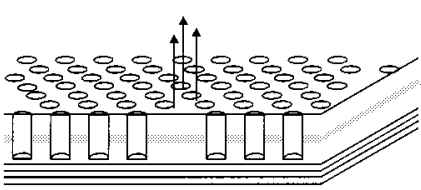

(b)

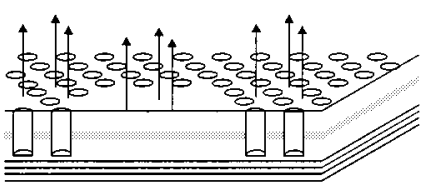

(d)
Fig. 14. Photonic Crystal Assisted RCLED. (a) 2-D PC etched through the active layer. (b) 2-D PC to inhibit lateral emission by creating an inplane cavity. (c) Shallow 2-D PC to extract guided mode, extraction of guided mode and active region coincidence. (d) 2-D PC surrounding active region to extract guided mode,extraction of guided mode and active region do not coincidence. The opposite mirror is in each case a DBR.

vented in the guided modes [64]. Eliminating the guided modes at the transition frequency, spontaneous emission can be enhanced to couple to the free space modes via the FP mode. In the first configuration, a 2-D PC is etched through the active layer of the planar RCLED [65]. Besides troublesome current injection, etching through the active layer can cause large nonradiative recombination rates due to induced surface states. An alternative configuration to somehow circumvent latter problem is sketched in (b). A bare cavity is surrounded by a 2-D PC. The high reflective 2-D PC creates an inplane cavity. To be efficient, the lateral dimensions of the microdisk have to be of the same order as the wavelength, $\mathrm{cf}$. the vertical dimensions of the planar RCLED. These dimensions are in general quite smaller than the diffusion length of carriers, and thus, do not fully tackle the nonradiative recombination problem at the etched surfaces. Moreover, due to the small inplane cavity dimensions, only small saturation values can be achieved [66]. Depending on spectral width and absorption losses configuration (a) or (b) is preferred [67]. Since a bandgap can be opened in the guided mode spectrum with holes not etched through the active material, surface recombination can be completely avoided using shallow gratings.

2) Extraction of the Guided Mode: Far more practical than inhibition of the guided mode is its extraction. The diffractive properties of the periodic grating can redirect the laterally propagating resonant guided mode to an extractable direction in the extraction cone. Because of the high power fraction in these guided modes (see Fig. 9), the use of such gratings can result in a higher $\eta_{\text {extr. }}$. In configuration (c), the shallow PC or grating coincidences with the active layer, but is not etched through it. The 2-D grating is designed to diffract efficiently the guided mode to the extraction cone, and thus, to the free space optical modes. Theoretical investigation of a GaAs $980-\mathrm{nm}$ cavity with GaAs-AlAs bottom DBR and metallic grating, the so called grating-assisted RCLED (GA-RCLED), predicts an $\eta_{\text {extr }}$ of over $43 \%$ [68]. First experiments confirm guided mode extraction, but $\eta_{\text {extr }}$ is still moderate [69]. A six-fold extraction enhancement is reported for an optically pumped asymmetric cavity with a DBR and a semiconductor-air interface [70], but absolute efficiencies are not mentioned.

A variant on the latter can be found in a 2-D periodic corrugation surrounding the active region. In these devices, the FP mode is extracted in the central part of the light source where the layers are homogeneous, the guided mode leaves the semiconductor in the surrounding periodically corrugated region. The region of generation and extraction of the guided mode are separated. The diameter of the unpatterned light generation region has to be smaller than the reabsorption length of the guided mode. The PC has to be designed to scatter the guided mode efficiently to the free space modes lying above the lightline, this for all inplane directions. Theoretical investigation of a top emitting laterally current injected $\lambda$-cavity with $\mathrm{AlO}_{x}-\mathrm{GaAs}$ DBR is promising: a supplementary extraction of $10 \%$ is predicted as worst case scenario [71]. Optically pumped thin-film devices showed a six-fold efficiency increase compared to the unetched thin-film in an NA $=0.7$ [72].

Configuration (c) is technologically more tedious since the grating, and thus, its deviations, influences the cavity directly (see Fig. 11 for sensitivity of $\eta_{\text {ext }}$ to cavity tuning variation). On the other hand, because the extraction and generation region are not separated, it is more efficient in the use of its active layer to total emitting surface than (d), resulting in a compact device with relative better $\eta_{\text {dens }}$.

\section{FUTURE PROSPECTS OF RCLEDS}

RCLEDs are not the only candidates that can be used as high-efficiency light source in communication and noncommunication applications. They compete with other high-efficiency LEDs and with VCSELs.

The key merit of a RCLED as compared to many other high-efficiency LED types is that it is a planar device, allowing for 1-D and 2-D arrays. In combination with its both high efficiency and high radiance or brightness, this makes the RCLED an ideal light source for multimode fiber coupling in array applications and opens the possibility of massively parallel optical data communication. Non-communication applications, e.g., sensors, printers, and scanners, do also require arrays of small diameter sources with high radiance.

Fabrication of a RCLED is similar to the fabrication of conventional planar LEDs: straightforward and at low cost. No advanced processing techniques are needed that can boost up costs.

In terms of modulation bandwidth, all LEDs are limited by the same fundamental limitation, i.e., the spontaneous emission lifetime. The modulation bandwidth of RCLEDs can be relatively high without penalty on efficiency because they generally have thin active layers and a small diameter resulting in a relatively high current density. Therefore, the carrier density in the active layer is relatively high leading to high modulation rates up to about $1 \mathrm{~Gb} / \mathrm{s}$ (see Section III.F and Section V).

While RCLEDs and LEDs both rely on spontaneous emission, VCSELs are based on stimulated emission in high $Q$ factor cavities. The cavity is formed by placing an active layer (QWs or QDs) between two DBRs. In contrast with the DBRs of the RCLED, both DBRs need to be highly reflecting, because the single-pass gain of these very thin active layers is rather small. This fundamentally different physical process of stimulated emission makes the VCSEL a monochromatic optical source, with a near-diffraction limited output field (single-mode VCSEL). These characteristics make the VCSEL 
far more suitable for coupling to single mode fibers than RCLEDs. Moreover, the fast stimulated emission makes it possible to modulate a biased VCSEL up to about $10 \mathrm{GHz}$.

On the other hand, fabrication of the RCLED is simpler and at lower cost than the VCSEL. Compared to a VCSEL with its high reflective DBRs, the small total thickness of the RCLED reduces the epitaxial growth time and the incorporated strain in case of lattice mismatch, simplifying wafer handling and device processing. Uniformity will be higher. For both VCSEL and RCLED, the principle is qualitatively suitable for all wavelengths, although quantitatively strongly depends on the appropriate material system. However, the RCLED has less stringent requirements on the material system. Especially the high gain in the active region and the high quality of the optical cavity are difficult the achieve in some material systems.

Another noteworthy aspect is that VCSELs, as all lasers, have a threshold current below which the efficiency is zero. LEDs do not have a threshold current and therefore they can outperform VCSELs in situations where a high efficiency is needed at a low power operation point. This can be the case in high density array applications.

The RCLED is an incoherent source. While this fact makes the RCLED unsuitable for coupling to single mode fibers, it should be emphasized that coherence is not necessary for coupling to multimode fibers. Even more, incoherence helps to reduce modal noise and speckle. Incoherence is even necessary for certain applications such as speckle-free medium-coherent sources for use in low-coherence interferometric techniques, to give one out of many possible examples.

Finally, the low-temperature dependence of the output power of a RCLED (see Section III.D) can be desirable in various applications that are used in extreme circumstances. However, due to the multifacetted and complex temperature behavior of both RCLEDs and VCSELs, a comparison can not be expressed in terms of simple statements.

In conclusion, it is clear that the RCLED can serve a broad range of low cost, high volume applications, both communication and noncommunication, and this for a broad range of wavelengths. The RCLED can be a favorable choice in comparison to VCSELs and most high-efficiency LEDs when: a relatively high radiance is needed, modulation bandwidth of $1 \mathrm{GHz}$ suffices and when the incoherent nature of the source is not a problem or is even an asset. In particular, dense array applications do profit from the combination of properties of the RCLED.

The RCLED has been introduced on the market for $650-\mathrm{nm}$ applications and is ready to be commercialized for a broader range of applications.

\section{REFERENCES}

[1] E. E. Loebner, "Subhistories of the light emitting diode," IEEE Trans. Electron. Dev., vol. ED-23, pp. 675-699, July 1976.

[2] H. Krömer, "A proposed class of heterojunction injection lasers," Proc. IEEE, vol. 51, p. 1782, 1963

[3] W. Lukosz and R. E. Kunz, "Light emission by magnetic and electric dipoles close to a plane interface. I total radiated power," J. Opt. Soc. Amer., vol. 67, no. 12, pp. 1607-1615, 1977.

[4] - "Light emission by magnetic and electric dipoles close to a plane interface. II radiation patterns of perpendicular oriented dipoles," J. Opt. Soc. Amer., vol. 67, no. 12, pp. 1615-1619, 1977.
[5] _ - "Light emission by magnetic and electric dipoles close to a plane interface. III radiation patterns of dipoles with arbitrary orientation," $J$. Opt. Soc. Amer., vol. 69, no. 11, pp. 1495-1503, 1979.

[6] W. Lukosz, "Theory of optical-environment-dependent spontaneous-emission rates for emitters in thin layers," Phys. Rev. B, vol. 22, no. 6, pp. 3030-3037, 1980.

[7] H. Yokoyama, K. Nishi, T. Anan, H. Yamada, S. D. Brorson, and E. P. Ippen, "Enhanced spontaneous emission from GaAs quantum wells in monolithic microcavities," Appl. Phys. Lett., vol. 57, no. 26, pp. 2814-2816, 1990.

[8] H. Yokoyama, "Physics and device applications of optical microcavities," Science, vol. 256, pp. 66-70, 1992.

[9] G. Bjork, S. Machida, Y. Yamamoto, and K. Igeta, "Modification of spontaneous emission rate in planar dielectric micro-cavity structures," Phys. Rev. A, vol. 44, no. 1, pp. 669-681, 1991.

[10] F. De Martini, M. Marrocco, P. Mataloni, L. Crescentini, and R. Loudon, "Spontaneous emission in the optical microscopic cavity," Phys. Rev. A, vol. 43, no. 5, pp. 2480-2497, 1991.

[11] D. G. Deppe and C. Lei, "Spontaneous emission from a dipole in a semiconductor micro-cavity," J. Appl. Phys., vol. 70, no. 7, pp. 3443-3448, 1991.

[12] Y. Yamamoto, S. Machida, and G. Bjork, "Micro-cavity semiconductor laser with enchanced spontaneous emission," Phys. Rev. A, vol. 44, no. 1, pp. 657-668, 1991.

[13] N. E. Hunt, E. F. Schubert, R. A. Logan, and G. J. Zydzik, "Enhanced spectral power density and reduced linewidth at $1.3 \mathrm{~mm}$ in an InGAaSP quantum well resonant-cavity light-emitting diode," Appl. Phys. Lett., vol. 61, no. 19, pp. 2287-2289, 1992.

[14] E. F. Schubert, Y.-H. Wang, A. Y. Cho, L.-W. Tu, and G. J. Zydzik, "Resonant cavity light-emitting diode," Appl. Phys. Lett., vol. 60, no. 8, pp. 921-923, 1992.

[15] E. Schubert, N. Hunt, M. Micovic, R. Malik, D. Sivco, A. Cho, and G. Zydzik, "Highly efficient light emitting diodes with microcavities," Science, vol. 265, pp. 943-945, 1994.

[16] J. Blondelle, H. De Neve, G. Borghs, P. Van Daele, P. Demeester, and R. Baets, "High efficiency $(>20 \%)$ micro-cavity light emitting diodes," Proc. IEE Coll. on Semiconductor Optical MicroCavity Devices and Photonic Bandgaps, 1996.

[17] H. De Neve, J. Blondelle, P. Van Daele, P. Demeester, R. Baets, and G. Borghs, "Recycling of guided mode light emission in planar micro-cavity light emitting diodes," Appl. Phys. Lett., vol. 70, no. 7, pp. 799-801, 1997.

[18] - Planar substrate-emitting micro-cavity light emitting diodes with $20 \%$ external QE, in SPIE Phot. West, San Jose, CA, 1997. presented.

[19] J. J. Wierer, D. A. Kellogg, and N. Holonyak, "Tunnel contact junction native-oxide aperture and mirror vertical-cavity surface-emitting lasers and resonant-cavity light-emitting diodes," Appl. Phys. Lett., vol. 74, no. 7, pp. 926-928, 1999.

[20] M. O. Holcomb, M. R. Krames, G. E. Hofler, C. Carter-Coman, E. Chen, P. Grillot, K. Park, N. F. Gardner, J.-W. Huang, J. Posselt, D. Collins, S. A. Stockman, G. M. Craford, F. A. Kish, I.-H. Tan, T. S. Tan, C. P. Kocot, and M. Hueschen, "High-power truncated-inverted-pyramid $\left(\mathrm{Al}_{x} \mathrm{Ga}_{1-x}\right)_{0.5} \mathrm{In}_{0.5} \mathrm{P}$ light-emitting diodes exhibiting $>50 \%$ external quantum efficiency," Appl. Phys. Lett., vol. 75, no. 16, pp. 2365-2367, 1999.

[21] I. Schnitzer, E. Yablonovitch, C. Carneau, T. J. Gmitter, and A. Scherer, " $30 \%$ external quantum efficiency from surface textured, thin-film lightemitting diodes," Appl. Phys. Lett., vol. 63, no. 16, pp. 2174-2176, 1993.

[22] R. Windisch, C. Rooman, S. Meinlschmidt, P. Kiesel, D. Zipperer, G. H. Doehler, B. Dutta, M. Kuijk, G. Borghs, and P. Heremans, "Impact of texture-enhanced transmission on high-efficiency surface-textured lightemitting diodes," Appl. Phys. Lett., vol. 79, no. 15, pp. 2315-2317, 2001.

[23] W. Schmid, M. Scherer, C. Karnutsch, A. Ploessl, W. Wegleiter, S. Schad, B. Neubert, and K. Streubel, "High efficiency, red and infrared light-emitting diodes using radial outcoupling taper," IEEE J. Select. Topics Quantum Electron., vol. 8, Mar.-Apr. 2002, to be published.

[24]

[25] S. Morikura, K. Kinoshita, K. Numata, and S. Furusawa, "High speed POF transmission technology and its standardization," in 27th European Conf. Optical Communication 2001, vol. 1, Amsterdam, Austria, pp. 20-21.

[26] C. Hooijer, G. Li, K. Allaart, and D. Lenstra, "Spontaneous emission in multilayer semiconductor structures," IEEE J. Quantum Electron., vol. 37, pp. 1161-1169, Sept. 2001.

[27] H. Benisty, H. De Neve, and C. Weisbuch, "Impact of planar microcavity effects on light extraction-Part I: Basic concepts and analytical trends," IEEE J. Quantum Electron., vol. 34, pp. 1612-1631, Sept. 1998. 
[28] — "Impact of planar microcavity effects on light extraction-Part II: Selected exact simulations and role of photon recycling," J. Quantum Electron., vol. 34, pp. 1632-1643, Sept. 1998.

[29] H. Benisty, R. Stanley, and M. Mayer, "Method of source terms for dipole emission modification in modes of arbitrary planar structures," J. Opt. Soc. Amer. A, vol. 15, no. 5, pp. 1192-1201, 1998.

[30] R. Baets, "Micro-cavity light emitting diodes," in Semiconductor Quantum Optoelectronics, A. Miller, M. Ebrahimzadeh, and D. M. Finlayson, Eds. St. Andrews, U.K., 1998.

[31] R. Baets, P. Bienstman, and R. Bockstaele, "Basics of dipole emission from a planar cavity," in Confined Photon Systems, Fundamentals and Applications, H. Benisty, J.-M. Gérard, R. Houdre, J. Rarity, and C. Weisbuch, Eds. New York: Springer-Verlag, 1999.

[32] J. Van Bladel, Electromagnetic Fields. New York: McGraw-Hill, 1964

[33] M. Yamanishi and I. Suemune, "Comment on polarization dependent momentum matrix elements in quantum well lasers," Jap. J. Appl. Phys., vol. 23, pp. L35-L36, 1984

[34] G. Jones, A. Ghiti, M. Silver, E. P. O'Reilly, and A. R. Adams, "Radiative perfomance of strained-layer lasers," in IEE Proc._J, vol. 140, Jan. 1993, pp. 85-89.

[35] J. M. Gérard and B. Gayral, "Strong purcell effect for InAs quantum boxes in three-dimensional solid-state microcavities," J. Lightwave Technol., vol. 17, pp. 2089-2095, Nov. 1999.

[36] E. M. Purcell, "Spontaneous emission probabilities at radiofrequencies," Phys. Rev., vol. 69, p. 681, 1946.

[37] G. Bjork, "On the spontaneous lifetime change in an ideal planar microcavity - Transition from a mode continuum to quantized modes," IEEE J. Quantum Electron., vol. 30, pp. 2314-2318, Oct. 1994.

[38] I. Abram, I. Robert, and R. Kuszelewicz, "Spontaneous emmission control in semiconductor microcavities with metallic or Bragg mirrors," IEEE J. Quantum Electron., vol. 34, pp. 71-76, Jan. 1998.

[39] P. Bienstman and R. Baets, "The RC2LED: An novel resonant-cavity LED design using a symmetric resonant cavity in the outcoupling reflector," IEEE J. Quantum Electron., vol. 36, pp. 669-673, June 2000.

[40] D. Ochoa, R. Houdré, R. P. Stanley, U. Oesterle, and M. Illegems, "Device simultaneous determination of the source and cavity parameters of a microcavity light-emitting diode," J. Appl. Phys., vol. 85, no. 5, pp. 2994-2996, 1999

[41] Z. Huang, C. C. Lin, and D. G. Deppe, "Spontaneous lifetime and quantum efficiency in light emitting diodes affected by a close metal mirror," IEEE J. Quantum Electron., vol. 29, no. 12, pp. 2940-2949, 1993.

[42] R. Bockstaele, "Resonant Cavity Light Emitting Diode Based Parallel Interconnections," Ph.D. Thesis, Ghent Univ., Ghent, Belgium, 2001.

[43] C. Weisbuch and B. Vinter, Quantum Semiconductor Structures-Fundamentals and Applications. San Diego, CA: Academic, 1991.

[44] R. Ram, D. Babic, R. York, and J. Bowers, "Spontaneous emission in microcavities with distributed mirros," IEEE J. Quantum Electron., vol. 31, pp. 399-410, Feb. 1995

[45] P. Yeh, Optical Waves in Layered Media. New York: Wiley, 1988.

[46] M. Rattier, H. Benisty, and C. Weisbuch, "Photonic crystal extractor," presented at the Electromagnetic Crystal Structures: Proceedings of Workshop on Photonic and Electromagnetic Crystal Structures III (PECS3), 9-14, June 2001, T. F. Krauss, Ed., St. Andrews, U.K., 2001.

[47] E. F. Schubert, N. E. J. Hunt, R. J. Malik, M. Micovic, and D. L. Miller, "Temperature and modulation characteristics of resonant-cavity lightemitting diodes," J. Lightwave Technol., vol. 14, pp. 1721-1729, July 1996.

[48] L. Coldren and S. Corzine, Diode Lasers and Photonic Integrated Circuits. New York: Wiley, 1995

[49] K. Tanaka, T. Nakamura, W. Takamatsu, M. Yamanishi, Y. Lee, and T. Ishihara, "Cavity-induced changes of spontaneous emission lifetime in one-dimensional semiconductor cavities," Phys. Rev. Lett., vol. 74, no. 17, pp. 3380-3386, 1995.

[50] R. Bockstaele, T. Coosemans, C. Sys, L. Vanwassenhove, A. Van Hove, B. Dhoedt, I. Moerman, P. Van Daele, R. G. Baets, R. Annen, H. Melchior, J. Hall, P. L. Heremans, M. Brunfaut, and J. Van Campenhout, "Realization and characterization of $8 \times 8$ resonant cavity LED arrays mounted onto CMOS drivers for POF-based interchip interconnections," J. Select. Topics Quantum Electron., vol. 5, pp. 224-235, Feb. 1999.

[51] P. Modak, M. D'Hondt, I. Moerman, P. Van Daele, P. Mijlemans, and P Demeester, "5.2\% efficiency InAlGaP microcavity LED's at $640 \mathrm{~nm}$ on Ge substrates," Electron. Lett., vol. 37, no. 6, pp. 377-378, 2001.

[52] R. Wirth, C. Karnutsch, S. Kugler, S. Thaler, and K. Streubel, "Red and orange resonant cavity LEDs," in Proc. SPIE Light-Emitting Diodes: Research, Manufacturing, and Applications V, vol. 4278, 2001.
[53] S. Orsila, T. Leinonen, P. Uusimaa, M. Saarinen, M. Guina, P. Sipila, V. Vilokkinen, P. Melanen, M. Dumitrescu, and M. Pessa, "Resonant cavity light-emitting diodes grown by solid source MBE," J. Cryst. Growth, vol. 227-228, pp. 346-351, 2001

[54] J. W. Gray, Y. S. Jalili, P. N. Stavrinou, M. Whitehead, G. Parry, A. Joel, R. Robjohn, R. Petrie, S. Hunjan, P. Gong, and G. Duggan, "Highefficiency, low voltage resonant-cavity light-emitting diodes operating around 650 nm," Electron. Lett., vol. 36, no. 20, pp. 1730-1731, 2000.

[55] R. Wirth, C. Karnutsch, S. Kugler, and K. Streubel, "High efficiency resonant cavity LED's emitting at $650 \mathrm{~nm}$," IEEE Photon. Technol. Lett., vol. 13, pp. 421-423, May 2001

[56] R. Bockstaele, J. Derluyn, C. Sys, S. Verstuyft, I. Moerman, P. Van Daele, and R. Baets, "Realization of highly efficient $850 \mathrm{~nm}$ top emitting resonant-cavity light-emitting diodes," Electron. Lett., vol. 35, no. 18 , pp. $1564-1565,1999$.

[57] P. Sipila, M. Saarinen, V. Vilokkinen, S. Orsila, P. Melanen, P. Savolainen, M. Toivonen, M. Dumitrescu, and M. Pessa, "Resonant cavity LED's at 655 and $880 \mathrm{~nm}$ wavelengths," in Proc. SPIE Light-Emitting Diodes: Research, Manufacturing, and Applications IV, vol. 3938, H. W. Yao, I. T. Ferguson, and E. F. Schubert, Eds., 2000, pp. 82-89.

[58] B. Depreter, I. Moerman, R. Baets, P. Van Daele, and P. Demeester, "InP based $1300 \mathrm{~nm}$ microcavity LED's with 9\% quantum efficiency," Electron. Lett., vol. 36, no. 15, pp. 1303-1304, 2000.

[59] M. Linnik and A. Christou, "High reflective Bragg mirrors for VCSEL application at 1.3 and $1.55 \mu \mathrm{m}$," in Proc. SPIE Phys. Simulation Optoelectron. Devices VIII, vol. 3944, H. Binder, P. Blood, and M. Osinski, Eds., 2000, pp. 874-881.

[60] M. C. Larson, C. W. Coldren, S. G. Spruytte, H. E. Petersen, and J. S. Harris, "Low-threshold oxide-confined GaInNA's long wavelength vertical cavity lasers," IEEE Photon. Technol. Lett., vol. 12, pp. 1598-1600, Dec. 2000.

[61] A. Fiore, U. Oesterle, R. P. Stanley, R. Houdre, F. Lelarge, M. Ilegems, P. Borri, W. Langbein, D. Birkedal, J. M. Hvam, M. Cantoni, and F. Bobard, "Structural and electrooptical characteristics of quantum dots emitting at $1.3 \mu \mathrm{m}$ on gallium arsenide," IEEE J. Quantum Electron., vol. 37, pp. 1050-1058, Aug. 2001

[62] B. Depreter, S. Verstuyft, I. Moerman, R. Baets, and P. Van Daele, "InP-based microcavity light emitting diodes emitting at $1.3 \mu \mathrm{m}$ and $1.55 \mu \mathrm{m}$," in Proc. 11th International Conf. InP and Related Materials (IPRM), Davos, Switzerland, May 16-20, 1999, pp. 227-230.

[63] F. Salomonsson, S. Rapp, K. Streubel, M. Hammar, and J. Daleiden, "InP-based $1.55 \mu \mathrm{m}$ resonant cavity light-emitting diode with two epitaxial mirrors," Physica Scripta, vol. T79, pp. 135-137, 1999.

[64] M. G. Salt, P. Andrew, and W. L. Barnes, "Microcavities, texture symmetry, and photonic bandgaps," J. Opt. Soc. Amer. B, vol. 18, no. 20, pp 240-243, 2001.

[65] S. Fan, P. R. Villeneuve, J. D. Joannopoulos, and E. F. Schubert, "High extraction efficiency of spontaneous emission from slabs of photonic crystals," Phys. Rev. Lett., vol. 78, no. 17, pp. 3294-3297, 1997.

[66] W. D. Zhou, J. Sabarinathan, P. Bhattacharya, B. Kochman, E. W. Berg, P.-C. Yu, and S. W. Pang, "Charactristics of a photonic bandgap single defect microcavity electroluminescent device," IEEE J. Quantum Electron., vol. 37, pp. 1153-1160, Sept. 2001.

[67] S. Fan, P. R. Villeneuve, and J. D. Joannopoulos, "Rate-equation analysis of output efficiency and modulation rate of photonic-crystal light-emitting diodes," IEEE J. Quantum Electron., vol. 36, pp. 1123-1130, Oct. 2000.

[68] D. Delbeke, P. Bienstman, R. Bockstaele, and R. Baets, "Rigorous electromagnetic analysis of dipole emission in periodically corrugated layers: The grating-assisted resonant-cavity light-emitting diode," $J$. Opt. Soc. Amer. A, May 2002. to be published.

[69] D. Delbeke, C. Sys, I. Moerman, P. Van Daele, and R. Baets, "Electrically pumped grating-assisted resonant-cavity light-emitting diodes," presented at the Light-Emitting Diodes: Research, Manufacturing, and Applications VI, Proc. SPIE, vol. 4641, 2002.

[70] A. A. Erchak, D. J. Ripin, S. Fan, P. Rakich, J. D. Joannopoulos, E P. Ippen, G. S. Petrich, and L. A. Kolodziejsk, "Enhanced coupling to vertical radiation using a two-dimensional photonic crystal in a semiconductor light-emitting diode," Appl. Phys. Lett., vol. 78, no. 5, pp. 563-565, 2001.

[71] M. Rattier, H. Benisty, and C. Weisbuch, "Photonic crystal extractor," presented at the Electromagnetic Crystal Structures: Proceedings of Workshop on Photonic and Electromagnetic Crystal Structures III (PECS3), 9-14 June 2001, T. F. Krauss, Ed., St. Andrews, U.K., 2001.

[72] M. Boroditsky, T. F. Krauss, R. Coccioli, R. Vrijen, R. Bhat, and E. Yablonovitch, "Light extraction from optically pumped light-emitting diode by thin-slab photonic crystal," Appl. Phys. Lett., vol. 75, no. 8, pp. 1036-1038, 1999. 


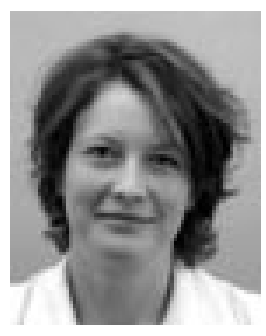

Danaë Delbeke received a degree in electrical engineering from Ghent University, Ghent, Belgium, in 1997. She is working toward the Ph.D. degree in electrical engineering at Ghent University.

Her research interests include modeling, design and fabrication of gratings, and grating-assisted resonant-cavity devices.

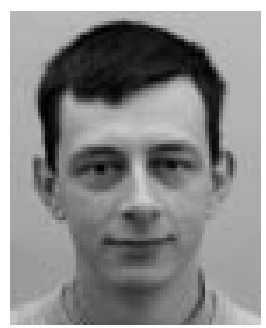

Ronny Bockstaele was born in Ghent, Belgium, in 1973. He received the degree in electrical engineering and $\mathrm{Ph}$.D. degree in electrical engineering on the study of resonant cavity light-emitting diodes (LEDs) for parallel optical interconnections from the University of Ghent, Belgium, in 1996 and 2001, respectively.

$\mathrm{He}$ is currently combining two jobs: one at the Department of Information Technology, University of Ghent, where he is involved in research on parallel optical interconnections, and one as an independent engineer.

He is a member of the Optical Society of America and the Lasers ElectroOptics Society (LEOS).

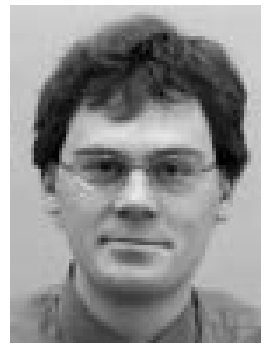

Peter Bienstman was born in Ghent, Belgium, in 1974. He received the degree in electrical engineering and the Ph.D. degree in the Department of Information Technology (INTEC) from Ghent University, Belgium, in 1997 and 2001, respectively.

His research interests include the modeling of optical structures, notably resonant-cavity light-emitting diodes, vertical-cavity surface-emitting lasers, and photonic crystal structures. He has published several papers and has one patent application pending.

Dr. Bienstman is a member of IEEE Lasers Electro-Optics Society.

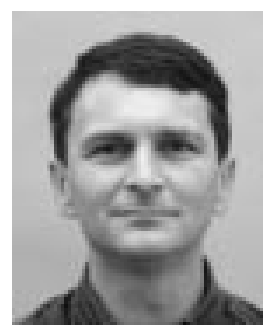

Roel Baets received the degree in electrical engineering from Ghent University, Belgium, the M.Sc. degree in electrical engineering from Stanford University, Stanford, CA, and the Ph.D. degree from Ghent University, Ghent, Belgium, in 1980, 1981, and 1984, respectively.

Since 1981, he has been with the Department of Information Technology (INTEC) of Ghent University. Since 1989, he is a professor in the engineering faculty of Ghent University. From 1990 to 1994, he has also been a part-time professor at the Technical University of Delft, The Netherlands. Roel Baets has mainly worked in the field of III-V devices for optoelectronic systems. With about 300 publications and conference papers he has made contributions to the design and fabrication of semiconductor laser diodes, passive guided wave devices, photonic integrated circuits (PICs) and microoptic components. He leads the Optoelectronic Components and Systems group at Ghent University-INTEC (which is an associated laboratory of IMEC), working on photonic devices for optical communication and optical interconnect.

Dr. Baets is a member of the Optical Society of America, IEEE Lasers Electro-Optics Society, SPIE, and the Flemish Engineers Association. He has been member of the program committees of OFC, ECOC, IEEE Semiconductor Laser Conference, ESSDERC, CLEO-Europe, and the European Conference on Integrated Optics. From 1999 to 2001, he was chairman of the IEEE-LEOS-Benelux chapter .

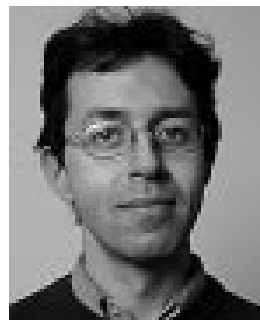

Henri Benisty received the Ph.D. degree from Ecole Normale Supérieure, Paris, France, working on accumulation layers at $\mathrm{Si}$ interfaces, in 1989.

Since then, he worked on semiconductor nanostructures with Thomson-LCR (now TRT Thales), Orsay, France, and is now teaching in Versailles University, Versailles, France. In 1994, he switched to the field of microcavities and photonic crystals. He has interest in two main kinds of applications of these systems: efficient and novel light sources, and integrated optics. 